\title{
Flow around an oscillating circular disk at low to moderate Reynolds numbers
}

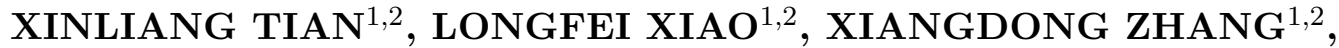 \\ JIANMIN YANG ${ }^{1,2} \dagger$, LONGBIN TAO ${ }^{3}$ AND DAN YANG ${ }^{4}$ \\ ${ }^{1}$ State Key Laboratory of Ocean Engineering, Shanghai Jiao Tong University, Shanghai \\ 200240, China \\ ${ }^{2}$ Collaborative Innovation Center for Advanced Ship and Deep-Sea Exploration, Shanghai \\ 200240, China \\ ${ }^{3}$ School of Marine Science and Technology, Newcastle University, Newcastle upon Tyne NE1 \\ 7RU, United Kingdom \\ ${ }^{4}$ School of Naval Architecture and Ocean Engineering, Huazhong University of Science and \\ Technology, Wuhan 430074, China
}

(Received ?; revised ?; accepted ?. - To be entered by editorial office)

Direct numerical simulations of the flow induced by a circular disk oscillating sinusoidally along its axis are performed. The aspect ratio ( $\chi=$ diameter/thickness) of the disk is 10 . The Reynolds number $(R e)$, based on the maximum speed and the diameter of the disk, is in the range of $50 \leqslant R e \leqslant 800$. The Keulegan-Carpenter number $(K C)$ is in the range of $1 \leqslant K C \leqslant 24$. Five flow regimes are observed in the considered $R e-K C$ space: (I) axisymmetric flow (AS), (II) planar symmetric flow in the low- $K C$ region (P$\mathrm{SL}$ ), (III) azimuthally rotating flow in the low- $K C$ region (ARL), (IV) planar symmetric flow in the high- $K C$ region (PSH) and $(\mathrm{V})$ azimuthally rotating flow in the high- $K C$ region (ARH). The critical boundaries between different flow regimes are identified based on the evolutions of the magnitude and direction of transverse force acting on the disk. For the non-axisymmetric flow regimes, the flow is one-sided with respect to the axis of the disk and is associated with a nonzero mean value of the transverse force acting on the disk.

Key words: flow-structure interactions, vortex instability, vortex interactions

\section{Introduction}

Interest in the flow around a circular disk goes back many years. Many experimental studies (see, e.g., Marshall \& Stanton 1931; Willmarth et al. 1964; Kuo \& Baldwin 1967; Roos \& Willmarth 1971; Roberts 1973; Berger et al. 1990; Fernandes et al. 2007; Zhong \& Lee 2012) and numerical calculations (see, e.g., Michael 1966; Rimon 1969; Rivet et al. 1988; Shenoy \& Kleinstreuer 2008; Fabre et al. 2008; Auguste et al. 2010; Chrust et al. 2010; Shenoy \& Kleinstreuer 2010; Yang et al. 2014a, 2015) addressing this issue have been reported. As a typical case of an axisymmetric body, studies on circular disks are often conducted and discussed together with another typical axisymmetric body, a sphere, which shares some similarities in the transition scenario (Fabre et al. 2008; Chrust et al. 2010).

The transition scenario in the wake of the uniform flow normal to a circular disk is

$\dagger$ Email address for correspondence: jmyang@sjtu.edu.cn 
dependent on both the Reynolds number and the aspect ratio of the disk. The aspect ratio of the disk is defined as $\chi=D / t_{d}$, where $D$ and $t_{d}$ are the diameter and thickness of the disk, respectively. A value with $\chi=\infty$ refers to an infinitely thin disk, which is sometimes called a "flat disk" in the literature. It has been reported that the flow is steady and axisymmetric at sufficiently low Reynolds numbers, corresponding to the "trivial state" (TS), as it has been named by Auguste et al. (2010). The first bifurcation occurs at the critical value $R e_{c 1}$, resulting in a reflectionally symmetric flow represented by a pair of steady streamwise vortices and a steady lift force. This state has been named a "steady state" (SS) by Fabre et al. (2008) and Meliga et al. (2009) and a "steady asymmetric state" by Shenoy \& Kleinstreuer (2008). According to direct numerical simulation (DNS) results for disks with $\chi=2,3,4,6$ and 10, the relation between the critical Reynolds number and the aspect ratio of the disk can be described as $R e_{c 1} \approx 116.5\left(1+\chi^{-1}\right)$ (Fernandes et al. 2007). For a flat disk $(\chi=\infty)$, a reasonable consensus that the value of $R e_{c 1}$ is between 115 and 117 has been achieved (Natarajan \& Acrivos 1993; Fabre et al. 2008; Meliga et al. 2009; Chrust et al. 2010). For a disk of $\chi=10, R e_{c 1}$ has been reported to be 135 by Shenoy \& Kleinstreuer (2008) and 129.6 by Chrust et al. (2010). A value of $R e_{c 1} \approx 159.4$ has been reported by Auguste et al. (2010) for a thicker disk with $\chi=3$.

A secondary Hopf bifurcation occurs as the Reynolds number increases further. The new state is characterized as a "three-dimensional periodic flow with regular rotation" (Shenoy \& Kleinstreuer 2008). Unlike in the case of the sphere, the lift force of the disk in this state is azimuthally oscillating. This state has also been referred to as a "YinYang" (YY) state by Auguste et al. (2010), a "mixed mode with phase $\pi$ " $\left(M M_{\pi}\right)$ by Meliga et al. (2009) and "reflectional symmetry breaking" (RSB) by Fabre et al. (2008). The critical Reynolds number for the secondary bifurcation $\left(R e_{c 2}\right)$ has been reported to lie between 121 and 125.6 for a flat disk $(\chi=\infty)$ (Fabre et al. 2008; Natarajan \& Acrivos 1993; Chrust et al. 2010) and between 136.3 and 138.7 (Chrust et al. 2010) or at approximately 155 (Shenoy \& Kleinstreuer 2008) for a disk with $\chi=10$.

As the Reynolds number increases still further, depending on the aspect ratio of the disk, the transition scenarios and the critical Reynolds numbers between different flow states become more complicated. Auguste et al. (2010) investigated the bifurcations in the wake of a disk with $\chi=3$. It was reported that at least four additional bifurcations are encountered before a chaotic state is reached, namely, the "Zig-zig" (Zz), "Knit-Knot" (KK), "Yin-Yang" (YY) and "Zig-Zag" (ZZ) modes. Chrust et al. (2010) conducted an extensive parametric study of the transition scenario in the wake of a disk with $\chi \geqslant 1$. A very detailed and systematic picture of the transition scenario was presented in the two-parameter space of the Reynolds number and the aspect ratio of the disk. A more detailed review is provided by Ern et al. (2012).

Apart from the studies of the flow around a fixed disk, the instabilities in the wake and path of a freely falling (or rising) disk under the action of gravity (or buoyancy) have attracted extensive attention. According to the experimental studies performed by Zhong \& Lee (2012), the wake transition scenarios are similar to those of the flow patterns behind a fixed disk. The transition from axisymmetry to plane symmetry was found at $R e_{c 1} \approx 105$, while the Hopf bifurcation occurred at $R e_{c 2} \approx 118$. Although such investigations on the first bifurcations in the wake of a fixed disk have indeed furthered the understanding of the mechanisms of the oscillatory motion of the freely moving disk, there is no doubt that the latter case is significantly more complicated. The problem of freely falling or rising bodies in a fluid at rest is governed by three control parameters: the geometrical parameter $\chi$, the body-to-fluid density ratio $\bar{\rho}$ and the Archimedes number $A r$. One of the widely accepted recognitions is that the presence of vorticity in the flow 
is the only source of path instability. However, this does not mean that the dynamics of the freely moving disk can be considered as that of a fixed disk. Fernandes et al. (2007) reported a delayed onset of instabilities for a freely rising disk compared with that of a fixed disk. This finding was later confirmed by Chrust et al. (2014). In other words, the freedom of the disk has a stabilizing effect on the onset of the primary bifurcation. Moreover, Auguste et al. (2013) noted that it was essentially incorrect to expect that the bifurcations for the falling body to closely mirror the wake of the same disk held fixed in a uniform stream. The dynamics of the wake and path of a freely moving disk are intrinsically coupled. Therefore, the stability analysis of this type of problem must consider the body + fluid system as fully coupled (Tchoufag et al. 2014).

Undoubtedly significant insight into the fluid dynamics of a disk has been achieved through these numerous experimental and numerical simulations. Nevertheless, there are still some aspects that are not fully understood and merit further investigation. With regard to the flow configurations, the steady flow over a fixed circular disk or a freely falling/rising circular disk in a stationary fluid were considered in the previous studies. In both of these cases, the flow relative to the disk is only in one direction, and the wake flow is always behind the disk. By contrast, the oscillatory flow over a circular cylinder or a circular cylinder oscillating in a quiescent fluid has been extensively studied (see, e.g., Bearman 1984; Williamson \& Govardhan 2008; An et al. 2011). Unfortunately, to the best of our knowledge, similar studies have not yet been performed for the circular disk. For a disk oscillating in a fluid at rest, the disk moves back and forth, accelerates and decelerates. Some new and interesting phenomena are expected to be revealed. This is the motivation of the present study, and four questions are to be answered: (i) what is the wake of the oscillating disk, (ii) how many types of flow regimes exist, (iii) what is the dependence of the threshold on the oscillating amplitude and Reynolds number, and (iv) what is the connection between the wake instabilities of a fixed disk and those of an oscillating disk.

With these questions, we start our study by considering a simplest case: an isolated circular disk that is forced to oscillate sinusoidally along its axis. Here, we introduce the coordinate system $(x, y, z)$, whose origin is located at the center of the disk. The $z$-axis of the coordinate system is parallel with the axis of the disk. The axial displacement of the disk is a function of time: $z_{d i s k}(t)=a \sin (2 \pi t / T)$, where $a$ is the amplitude of the oscillatory motion, $T$ is the period of oscillation, and $t$ is the time. Therefore, the axial velocity of the disk is taken as $u_{z}(t)=U_{\max } \cos (2 \pi t / T)$, where $U_{\max }$ is the maximum speed of the disk, i.e., $U_{\max }=2 \pi a / T$.

It has been reported that the aspect ratio of the disk plays a significant role in determining the transition process and vortex structure in the wake of a steady flow (Shenoy \& Kleinstreuer 2010). Therefore, the key dimensionless parameters that define the problem are the Reynolds number $(R e)$, the Keulegan-Carpenter number $(K C)$ and the disk aspect ratio $\chi$. The respective definitions of $R e$ and $K C$ are given as follows:

$$
\begin{gathered}
R e=\frac{U_{\max } D}{\nu} \\
K C=\frac{U_{\max } T}{D}=\frac{2 \pi a}{D}
\end{gathered}
$$

where $\nu$ is the kinematic viscosity of the fluid.

It is noteworthy that, according to the study for the transition scenario in the wake of a fixed disk by Chrust et al. (2015), the supporting structure is one of the main sources resulting in the discrepancies between the numerical and experimental results. The flow regimes in the present oscillating case may also be influenced by the supporting 
structure. In the present study, we attempted to reveal the fundamental fluid dynamics of an oscillating circular disk. Therefore, the supporting structure is not considered. In this case, numerical method is a more appropriate technique because the very ideal conditions could be achieved easily in numerical simulations.

The DNS method was used to calculate the flow around the disk, and the deforming mesh technique was employed to simulate the oscillatory motion of the disk. The ranges of $R e$ and $K C$ considered in the present study are $50 \leqslant R e \leqslant 800$ and $1 \leqslant K C \leqslant 24$, respectively. The disk aspect ratio considered in this study is 10 , which can be reasonably regarded as representative of a "thin" disk (Chrust et al. 2010). Another reason for the selection of an aspect ratio of 10 is for convenience in comparing the obtained results with the DNS results reported by Shenoy \& Kleinstreuer (2008).

The remainder of this paper is organized as follows. Details of the numerical simulations are presented in $\S 2$. The results are described in $\S 3$. Finally, a few concluding remarks are offered in $\S 4$.

\section{Numerical simulations}

\subsection{Mathematical formulations and numerical methods}

We consider a rigid circular disk that is forced to oscillate along its axis in a Newtonian incompressible fluid at rest. The Cartesian coordinate system $(x, y, z)$ is used in the present study. If these coordinates are labeled as $\left(x_{1}, x_{2}, x_{3}\right)$ and the velocity component in the $x_{i}$ direction is denoted by $u_{i}$, where $i=1-3$, then the Navier-Stokes (N-S) equations can be expressed as follows:

$$
\begin{gathered}
\frac{\partial u_{i}}{\partial x_{i}}=0 \\
\frac{\partial u_{i}}{\partial t}+u_{j} \frac{\partial u_{i}}{\partial x_{j}}=-\frac{1}{\rho} \frac{\partial p}{\partial x_{i}}+\nu \frac{\partial^{2} u_{i}}{\partial x_{j} \partial x_{j}}
\end{gathered}
$$

where $p$ is the pressure and $\rho$ is the density of the fluid. For clarity, the velocity components $u_{1}, u_{2}$ and $u_{3}$ are also denoted by $u_{x}, u_{y}$ and $u_{z}$, respectively.

The N-S equations were discretized using the finite volume method (FVM) based on the open-source computational fluid dynamics (CFD) code OpenFOAM. OpenFOAM is primarily applied for solving problems in continuum mechanics. It is built based on the tensorial approach and object-oriented techniques (Weller et al. 1998). The PressureImplicit with Splitting of Operators (PISO) scheme (pisoFoam) was used in the present study. The spatial schemes used for interpolation, gradient, Laplacian and divergence calculations were the linear, Gauss linear, Gauss linear corrected and Gauss linear schemes, respectively. All these schemes were of second order. The second-order Crank-Nicholson scheme was used for the time integration. Further details of these schemes are provided in OpenFOAM (2009).

The non-dimensional force coefficients in three directions, i.e., the $x, y$ and $z$ directions, are defined as follows:

$$
\left(C_{x}, C_{y}, C_{z}\right)=\frac{\left(F_{x}, F_{y}, F_{z}\right)}{\frac{1}{8} \rho U_{r e f}^{2} \pi D^{2}}
$$

where $F_{x}, F_{y}$ and $F_{z}$ are the force components acting on the disk in the $x, y$ and $z$ directions, respectively, and they are directly calculated by integrating the pressure and viscous shear stress over the disk surface; in the case of the analysis of the steady flow over a fixed disk performed for code validation (see $\S 2.2)$, the reference speed $\left(U_{\text {ref }}\right)$ is 


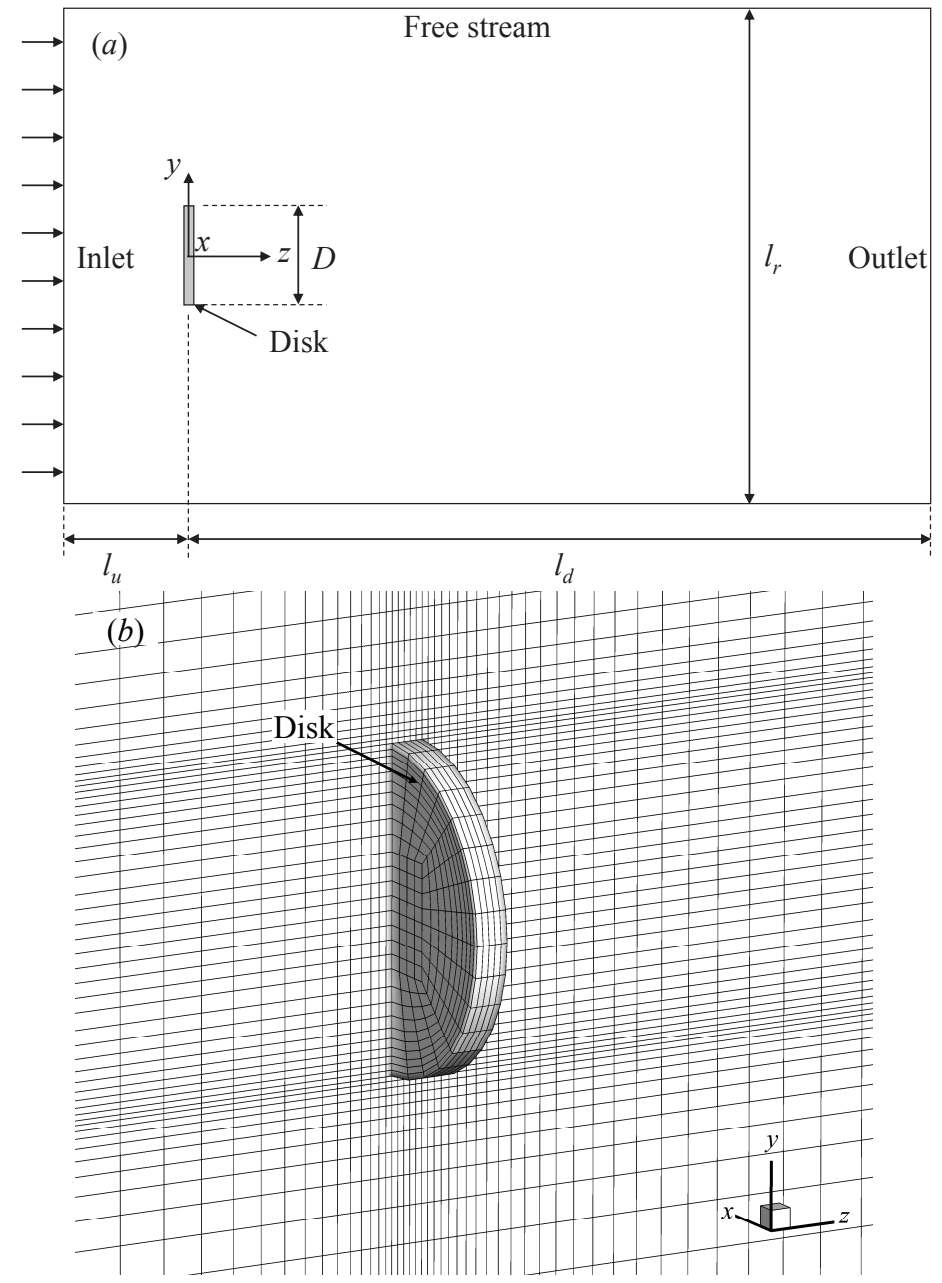

FiguRE 1. Stationary disk: (a) computational domain and boundary conditions (not to scale) and $(b)$ grid structures near the disk surface.

the free stream speed $\left(U_{s}\right)$, and in the case of the oscillating disk, $U_{\text {ref }}$ is the maximum speed of the disk $\left(U_{\max }\right)$.

The so-called $Q$-criterion proposed by Hunt et al. (1988) and the vorticity component in the $z$ direction $\left(\omega_{z}\right)$ are used to identify the vortical structures around the disk. $Q$ is defined as

$$
Q=-\frac{1}{2}\left(\|\boldsymbol{S}\|^{2}-\|\boldsymbol{\Omega}\|^{2}\right)
$$

where $\boldsymbol{S}$ and $\boldsymbol{\Omega}$ denote the strain and the rotation tensor, respectively.

\subsection{Code validation}

To validate the adopted numerical approach, benchmark calculations of the steady flow normal to a stationary disk are performed. Following the setup presented by Shenoy \& Kleinstreuer (2008), the aspect ratio of the disk is considered as $\chi=10$. A cylindrical computational domain with a cross section of diameter $l_{r}$ is used; see figure $1(a)$. The origin of the coordinate system $(x, y, z)$ is located at the center of the disk, and the $z$-axis coincides with the axis of the disk. The distances from the inlet and outlet boundaries to 


\begin{tabular}{|c|c|c|c|c|c|c|c|c|c|c|c|c|}
\hline & \multirow[b]{2}{*}{ Case } & \multirow[b]{2}{*}{ Elements } & \multirow[b]{2}{*}{$n_{1} / D$} & \multirow{2}{*}{$\begin{array}{c}\cdot \\
\Delta t U_{s} / D\end{array}$} & \multicolumn{3}{|c|}{ Domain size } & \multicolumn{5}{|c|}{$R e_{s}$} \\
\hline & & & & & $l_{u} / D$ & $l_{d} / D$ & $l_{r} / D$ & 10 & 30 & 50 & 75 & 100 \\
\hline \multirow{5}{*}{$C_{z}$} & S1 & 107520 & 0.02 & 0.01 & 5 & 30 & 24 & 4.00 & 2.15 & 1.70 & 1.43 & 1.28 \\
\hline & $\mathrm{S} 2$ & 365184 & 0.0133 & 0.0075 & 5 & 30 & 24 & 4.02 & 2.16 & 1.71 & 1.44 & 1.28 \\
\hline & S3 & 860160 & 0.01 & 0.005 & 5 & 30 & 24 & 4.03 & 2.16 & 1.70 & 1.44 & 1.28 \\
\hline & $\mathrm{S} 4$ & 735360 & 0.01 & 0.005 & 4 & 24 & 18 & 4.06 & 2.18 & 1.72 & 1.45 & 1.29 \\
\hline & S5 & 599040 & 0.01 & 0.005 & 2.5 & 15 & 12 & 4.21 & 2.25 & 1.78 & 1.50 & 1.34 \\
\hline \multirow{5}{*}{$L_{w} / D$} & S1 & 107520 & 0.02 & 0.01 & 5 & 30 & 24 & 0.40 & 0.87 & 1.24 & 1.60 & 1.88 \\
\hline & $\mathrm{S} 2$ & 365184 & 0.0133 & 0.0075 & 5 & 30 & 24 & 0.40 & 0.88 & 1.25 & 1.62 & 1.90 \\
\hline & S3 & 860160 & 0.01 & 0.005 & 5 & 30 & 24 & 0.41 & 0.89 & 1.26 & 1.63 & 1.91 \\
\hline & $\mathrm{S} 4$ & 735360 & 0.01 & 0.005 & 4 & 24 & 18 & 0.41 & 0.89 & 1.26 & 1.63 & 1.91 \\
\hline & S5 & 599040 & 0.01 & 0.005 & 2.5 & 15 & 12 & 0.41 & 0.90 & 1.27 & 1.64 & 1.93 \\
\hline
\end{tabular}

TABLE 1. Results for the axial drag coefficient $\left(C_{z}\right)$ and the recirculation length $\left(L_{w}\right)$ obtained for cases of different configurations for a stationary disk.
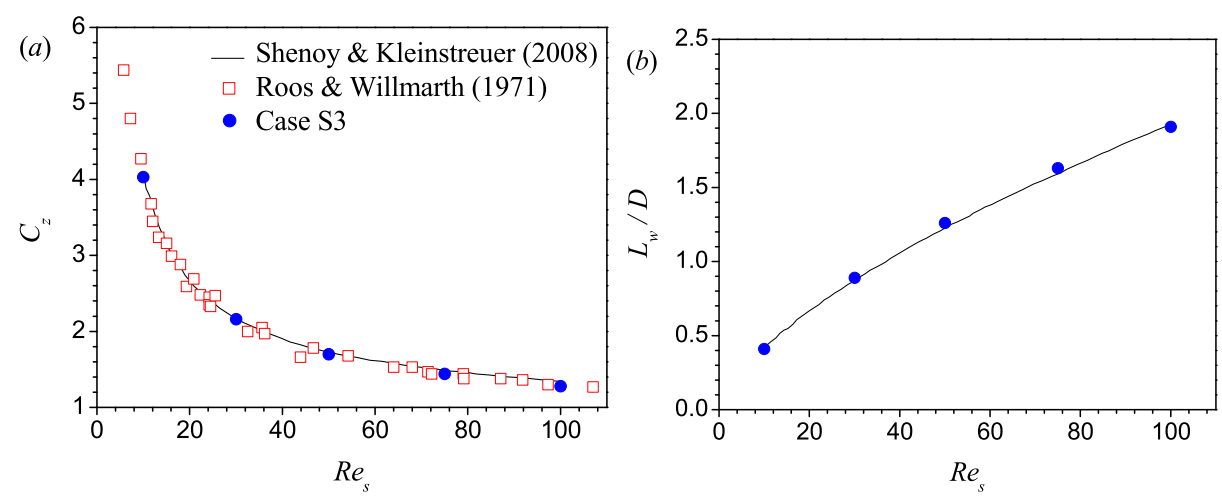

Figure 2. Comparisons of the results for the steady flow normal to a circular disk: $(a)$ axial drag coefficient $\left(C_{z}\right)$ and $(b)$ recirculation length $\left(L_{w}\right)$.

the center of the disk are denoted as $l_{u}$ and $l_{d}$, respectively. On the disk surface, no-slip and zero-normal-pressure-gradient boundary conditions are imposed. At the inlet boundary, a uniform velocity $\left(0,0, U_{s}\right)$ and zero-normal-pressure-gradient boundary conditions are prescribed. At the outlet boundary, the velocity is set to a zero normal gradient and the pressure is fixed at zero. At the free stream boundary, free-slip and zero-normalgradient boundary conditions are applied for the velocity and pressure, respectively. The topology of the mesh also follows that used in Shenoy \& Kleinstreuer (2008), and the computational domain is discretized with hexahedral elements; see figure $1(b)$. In the vicinity of the disk, the grids are finer to better resolve the steep gradient in this region. To assess the effects of the spatial and temporal resolutions on the results, three cases with different spatial and temporal resolutions are considered; see cases S1-S3 in table 1, where $n_{1}$ denotes the element size in the wall-normal direction near the disk surface, $\Delta t$ is the time step used in the simulations, $R e_{s}$ denotes the Reynolds number based on the free stream velocity $\left(U_{s}\right)$ and the disk diameter $(D)$, and $L_{w}$ denotes the recirculation length, which is defined as the streamwise distance from the center of the disk to the position where the streamwise velocity changes sign from negative to positive. In the considered Reynolds number region (i.e., $R e_{s}<R e_{c 1}$ ), the flow is steady and axisymmetric 


$\begin{array}{cccc}\text { Case } & & & \\ \text { present (S1) } & \chi & R e_{s} & S t \\ \text { present (S2) } & 10 & 160 & 0.118 \\ \text { present (S3) } & 10 & 160 & 0.115 \\ \text { present (S4) } & 10 & 160 & 0.114 \\ \text { present (S5) } & 10 & 160 & 0.115 \\ \text { present (S1) } & 10 & 180 & 0.120 \\ \text { present (S2) } & 10 & 180 & 0.120 \\ \text { present (S3) } & 10 & 180 & 0.117 \\ \text { present (S4) } & 10 & 180 & 0.116 \\ \text { present (S5) } & 10 & 180 & 0.122 \\ \text { Shenoy \& Kleinstreuer (2008) } & 10 & 160 & 0.113 \\ \text { Shenoy \& Kleinstreuer (2008) } & 10 & 180 & 0.113 \\ \text { Chrust et al. (2010) } & 10 & (136.3,138.7) & 0.115 \\ \text { Chrust et al. (2010) } & 10 & 154.4 & 0.114 \\ \text { Yang et al. (2014c) } & 5 & 152 & 0.113 \\ \text { Yang et al. (2014c) } & 5 & 172 & 0.113\end{array}$

TABLE 2. Comparisons of the Strouhal number for a stationary disk obtained in different studies.

(Auguste et al. 2010). As shown in table 1, only very small variations are observed in the results for $C_{z}$ and $L_{w}$ calculated for the different spatial and temporal resolutions, i.e., for cases S1-S3. To further assess the effects of the size of the computational domain on the calculated results, two more cases are conducted with a reduced computational domain size, see cases S4 and S5 in table 1. The meshes used in cases S3-S5 are designed to be of an equivalent density. As shown in table 1, a good convergence is observed in the results of $C_{z}$ and $L_{w}$ for cases S3-S5. It is concluded that the effects of the size of the computational domain on the calculated results are negligible for case S3. Finally, the axial drag coefficient $\left(C_{z}\right)$ and recirculation length $\left(L_{w}\right)$ obtained for case S3 are in good agreement with the DNS results reported by Shenoy \& Kleinstreuer (2008) and the experimental measurements reported by Roos \& Willmarth (1971); see figure 2.

To validate the capability of the present numerical approach for computing the unsteady wake flow, numerical simulations are conducted for Reynolds numbers of $R e_{s}=$ 160 and 180. As shown in table 2, the Strouhal number $S t$ represents the wake frequency nondimensionalized with the free stream velocity $\left(U_{s}\right)$ and the diameter of the disk $(D)$. Similar to the validation studies for the steady cases shown in table 1, the effects of the grid resolution, time step and computational domain size are also evaluated. The configurations of cases $\mathrm{S} 1-\mathrm{S} 5$ are kept the same as those shown in table 1. It is observed that the results of $S t$ in the present converged case S3 agree well with the results obtained in the literatures (Shenoy \& Kleinstreuer 2008; Chrust et al. 2010; Yang et al. 2014c).

Overall, the present numerical approach yields reliable results for the flow over a circular disk. Moreover, this numerical approach has previously been successfully applied to calculate the incompressible flow around an oscillating disk (Yang et al. 2014b).

\subsection{Computational domain and boundary conditions}

A spherical computational domain of radius $R_{\text {domain }}$ was used for the oscillating disk; see figure $3(a)$. The origin of the coordinate system is located at the center of the computational domain, and the $z$-axis coincides with the axis of the disk. The mean position of the disk is at the center of the computational domain. On the disk surface, the noslip boundary condition is imposed for the velocity; the pressure is set to a zero normal 


\begin{tabular}{|c|c|c|c|c|c|c|c|c|c|}
\hline \multirow[t]{2}{*}{ Case } & \multirow[t]{2}{*}{ Elements } & \multirow[t]{2}{*}{$n_{1} / D$} & \multirow[t]{2}{*}{$R_{\text {domain }}$} & \multirow[t]{2}{*}{$T / \Delta t$} & \multicolumn{2}{|c|}{$\begin{aligned} R e & =245 \\
K C & =24.5\end{aligned}$} & \multicolumn{2}{|c|}{$\begin{array}{l}R e=600 \\
K C=15\end{array}$} & $\begin{array}{l}R e=900 \\
K C=7.5\end{array}$ \\
\hline & & & & & $C_{D}$ & $C_{M}$ & $C_{D}$ & $C_{M}$ & $C_{M}$ \\
\hline O1 & 117760 & 0.0055 & $20 D$ & 5000 & 1.48 & 14.82 & 1.51 & 14.07 & 1.8411 .13 \\
\hline $\mathrm{O} 2$ & 397440 & 0.0036 & $20 D$ & 10000 & 1.42 & 17.15 & 1.44 & 14.07 & $\begin{array}{ll}1.78 & 11.08\end{array}$ \\
\hline O3 & 942080 & 0.0027 & $20 D$ & 15000 & 1.42 & 17.15 & 1.44 & 13.83 & 1.7611 .23 \\
\hline O4 & 457920 & 0.0036 & $30 D$ & 10000 & 1.43 & 17.00 & 1.46 & 13.87 & 1.7811 .11 \\
\hline
\end{tabular}

TABLE 3. Results for the axial drag coefficient $\left(C_{z}\right)$ and the recirculation length $\left(L_{w}\right)$ obtained for cases of different spatial and temporal resolutions for an oscillating disk.

gradient. At the outer boundary, the velocity is set to a zero normal gradient and the pressure is fixed at zero. It has been checked carefully that the effects of the boundary condition at the outer boundary on the calculated results are negligible in the present configuration. The entire computational domain is discretized with hexahedral elements, and the grid near the disk surface is of finer resolution to resolve the steep gradient in this region; see figure 3.

The oscillatory motion of the disk was realized using the deforming mesh method, in which the topology of the mesh does not change throughout the simulation. The mesh was updated at each time step by moving the grid points based on the permanent static mesh with $z_{d i s k}=0$, which was established at the beginning of the simulation (see figure $3 a$ as an example). To preserve the validity and quality of the mesh, a grid-smoothing method was applied, which is described as follows. Let $\boldsymbol{r}^{i}(t)$ represent the coordinates of point $i$ at time $t$, and let $\boldsymbol{r}_{d i s k}(t)=\left(0,0, z_{d i s k}\right)$ represent the displacement of the disk at time $t$; then, $\boldsymbol{r}^{i}(t)$ can be determined using the following equation:

$$
\boldsymbol{r}^{i}(t)=\boldsymbol{r}^{i}(0)+\boldsymbol{r}_{d i s k}(t) f^{*}
$$

The scaling function $f^{*}$ in equation $(2.5)$ is determined by the distance of the grid point from the center of the disk as follows:

$$
f^{*}= \begin{cases}1 & \text { if }\left|\boldsymbol{r}^{i}(0)\right| \leqslant R_{1} \\ 0.5 \sin \left[\pi\left(\frac{\left|\boldsymbol{r}^{i}(0)\right|-R_{2}}{R_{1}-R_{2}}-0.5\right)\right]+0.5 & \text { if } R_{1}<\left|\boldsymbol{r}^{i}(0)\right|<R_{2} \\ 0 & \text { if }\left|\boldsymbol{r}^{i}(0)\right| \geqslant R_{2}\end{cases}
$$

Here, the values of $R_{1}$ and $R_{2}$ in equation (2.6) are taken to be $R_{1}=2.5 D$ and $R_{2}=$ $R_{\text {domain }}$, respectively, where $R_{\text {domain }}$ is the radius of the spherical computational domain. Using the scaling function $f^{*}$, a smooth grid deformation can be obtained between the disk and the outer boundary. Thus, a good mesh quality can always be maintained during a simulation. Figure $3(b)$ shows an example of the grid structures at the maximum amplitude considered in this study, i.e., $z_{\text {disk }}=3.82 \mathrm{D}$ at $K C=24$.

\subsection{Convergence studies}

To assess the effects of the spatial and temporal resolutions on the calculated results, three cases with different numbers of grid elements and time steps, i.e., cases $\mathrm{O} 1-\mathrm{O} 3$ in table 3, are considered. For each case, the results for three different flow configurations, i.e., $(R e, K C)=(245,24.5),(600,15)$ and $(900,7.5)$, are presented. The parameters $C_{D}$ and $C_{M}$ shown in table 3 are the drag and inertia coefficients, respectively, which are calculated by splitting the axial force $\left(F_{z}\right)$ into drag and inertial terms following the 
(a)

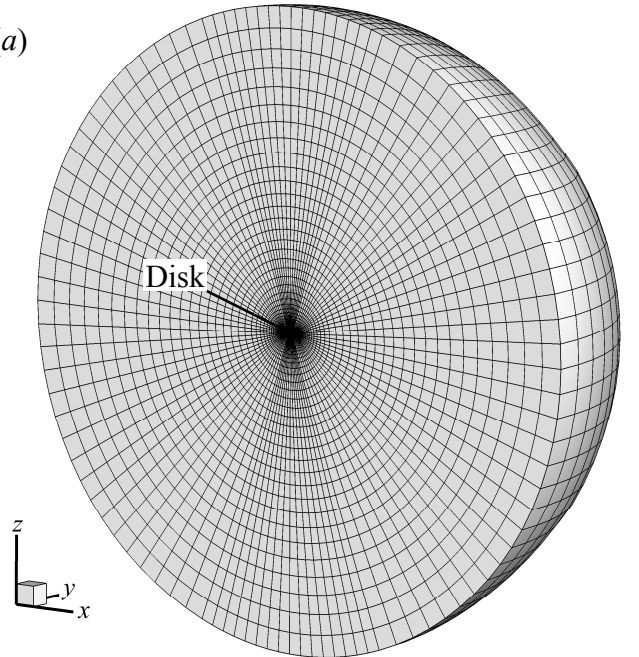

(b)

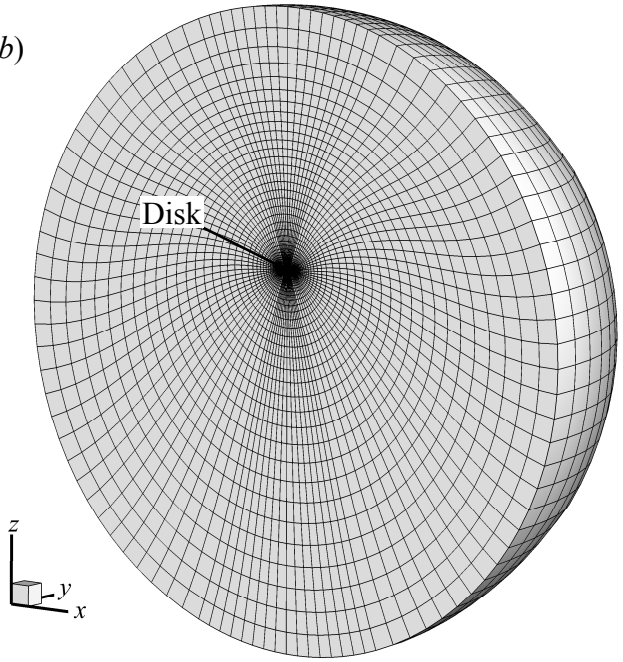

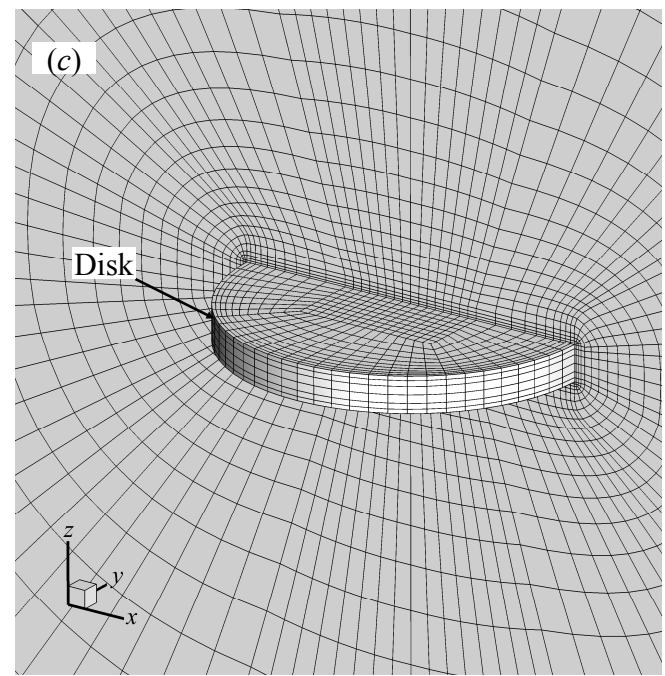

FiguRe 3. Oscillating disk: cut-away views of the computational domain and grid structures $(a)$ at the mean disk position $z_{\text {disk }}=0,(b)$ at the maximum disk displacement $\left(z_{\text {disk }} \approx 3.82 D\right.$ at $K C=24$ as an example) and $(c)$ in a detailed zoom of the region near the disk surface.

Morison equation (Morison et al. 1950), which states that

$$
F_{z}=-C_{D} \rho \frac{\pi}{8} D^{2} u_{z}(t)\left|u_{z}(t)\right|-C_{M} \rho \frac{\pi}{4} t_{d} D^{2} \dot{u}_{z}(t)
$$

In this study, $C_{D}$ and $C_{M}$ were calculated using the least-squares method. As shown in table 3, for all three considered flow configurations, the results of $C_{D}$ and $C_{M}$ obtained in cases $\mathrm{O} 2$ and $\mathrm{O} 3$ agree well with each other, indicating reasonable convergence with increasing spatial and temporal resolutions. The effects of the size of the computational domain on the calculated results are evaluated by considering a larger domain of radius $R_{\text {domain }}=30 D$; see case $\mathrm{O} 4$ in table 3 . Case O4 corresponds to a similar spatial resolution and the same time step in case $\mathrm{O} 2$. The results for $C_{D}$ and $C_{M}$ obtained in case $\mathrm{O} 4$ agree well with those in cases $\mathrm{O} 2$ and $\mathrm{O} 3$, indicating that a computational domain of radius $R_{\text {domain }}=20 D$ is sufficient to eliminate the effects of the outer boundary on the calculated results. 

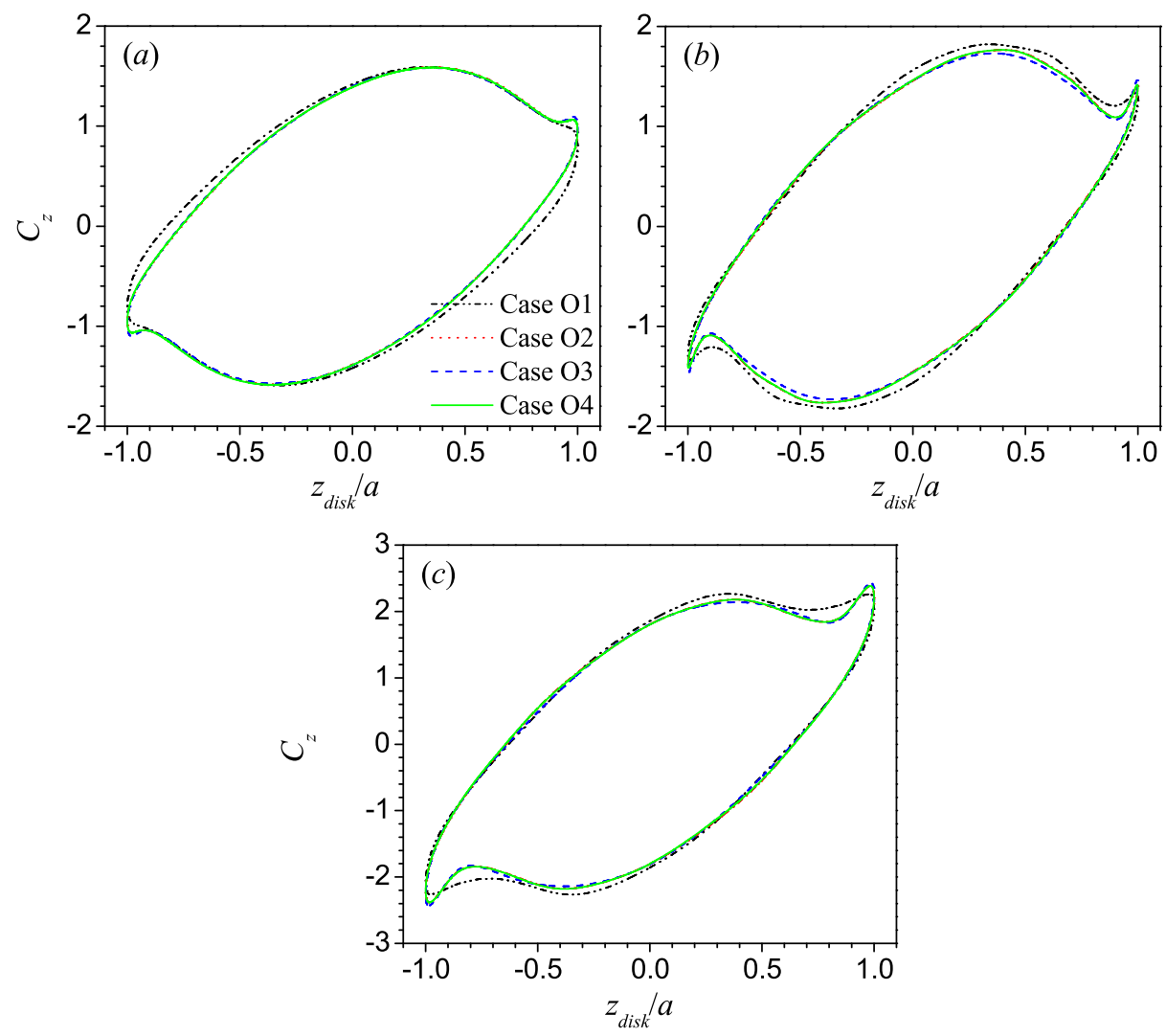

Figure 4. Axial drag coefficient $\left(C_{z}\right)$ with respect to the position of the disk $\left(z_{\text {disk }}\right)$ at $(a)$ $R e=245$ and $K C=24.5,(b) R e=600$ and $K C=15$ and $(c) R e=900$ and $K C=7.5$.

Moreover, the time traces of $C_{z}$ for the three considered $(R e, K C)$ configurations are plotted against $z_{\text {disk }}$ in figure 4 . Good consistency is found among the results of cases $\mathrm{O} 2-\mathrm{O} 4$; this again demonstrates that the effects of the spatial and temporal resolutions and of the size of the computational domain on the calculated results are negligible in the present study. In the following, all reported simulations were performed using the setup used in case O2, i.e., with 397440 grid elements, a time step of $T / 10000$ and a computational domain of radius $20 D$.

\section{Results and discussion}

According to the DNS studies performed by Shenoy \& Kleinstreuer (2008) concerning the uniform flow normal to a circular disk of $\chi=10$, the flow is steady and axisymmetric about the axis of the disk when $R e<135$. As the Reynolds number increases, regular bifurcation with a loss of azimuthal symmetry occurs. Similarly, for the oscillating disk considered in the present study, non-axisymmetric bifurcation may occur as $R e$ and $K C$ vary. Here, the flow regimes around a circular disk of $\chi=10$ are investigated in the considered $R e-K C$ space, i.e., for $50 \leqslant R e \leqslant 800$ and $1 \leqslant K C \leqslant 24$.

Numerical simulations were performed for 151 pairs of $R e$ and $K C$ values represented by solid dots in figure 5 . Preliminary inspection on the calculated flow field indicates that there are at least three types of flow regimes in the considered $R e-K C$ space, i.e, one 


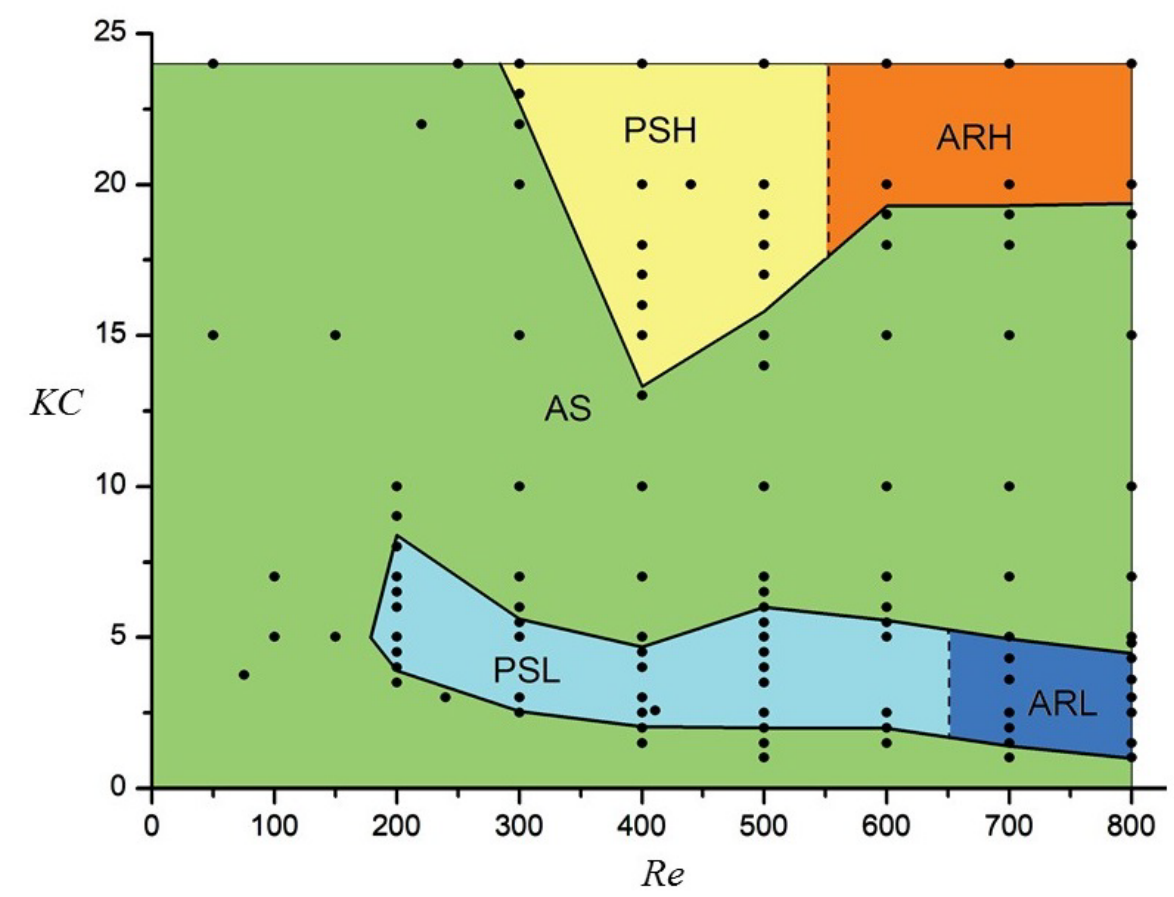

FiguRE 5. The critical boundaries between different flow regimes in $R e-K C$ space.

axisymmetric flow region in the middle and two non-axisymmetric flow regions in the lowand high- $K C$ regions. It is noteworthy that the onset of non-axisymmetry is not always clear-cut. The magnitude of the transverse force acting on the disk is closely related to the level of non-axisymmetry of the flow. Here, the magnitude of the transverse force coefficient is calculated as $C_{x y}=\sqrt{C_{x}^{2}+C_{y}^{2}}$. Close to the threshold of the axisymmetric and non-axisymmetric regimes, any perturbation is either exponentially amplified or damped. Therefore, the accurate threshold is determined by linear interpolation of the growth rate of $C_{x y}$. The details of the procedures used to determine the critical value of $R e$ and $K C$ corresponding to the axisymmetric and non-axisymmetric bifurcations are given in Appendix A. The two solid lines in figure 5 represent the threshold between axisymmetric and non-axisymmetric regimes. Further careful examination reveals that the non-axisymmetric flow begins to rotate around the axis of the disk as the Reynolds number increases. The boundary between the rotating and non-rotating flow regimes is identified by the dashed lines in figure 5 . Finally, five flow regimes in the considered $R e-$ $K C$ space are revealed here: (I) axisymmetric flow (AS), (II) planar symmetric flow in the low- $K C$ region (PSL), (III) azimuthally rotating flow in the low- $K C$ region (ARL), (IV) planar symmetric flow in the high- $K C$ region $(\mathrm{PSH})$ and $(\mathrm{V})$ azimuthally rotating flow in the high- $K C$ region $(\mathrm{ARH})$. In the following, we select one example case from each flow regime and present the corresponding force coefficients and flow visualizations.

\subsection{Regime I: Axisymmetric flow (AS)}

Figure 6 shows the time traces of the force coefficients for the AS regime, where $R e=600$ and $K C=15$. As shown, a periodic variation in $C_{z}$ is observed, indicating a welldeveloped flow. However, the transverse force coefficients $C_{x}$ and $C_{y}$ are zero.

Figure 7 shows the three-dimensional vortical structures identified based on the $Q$ - 


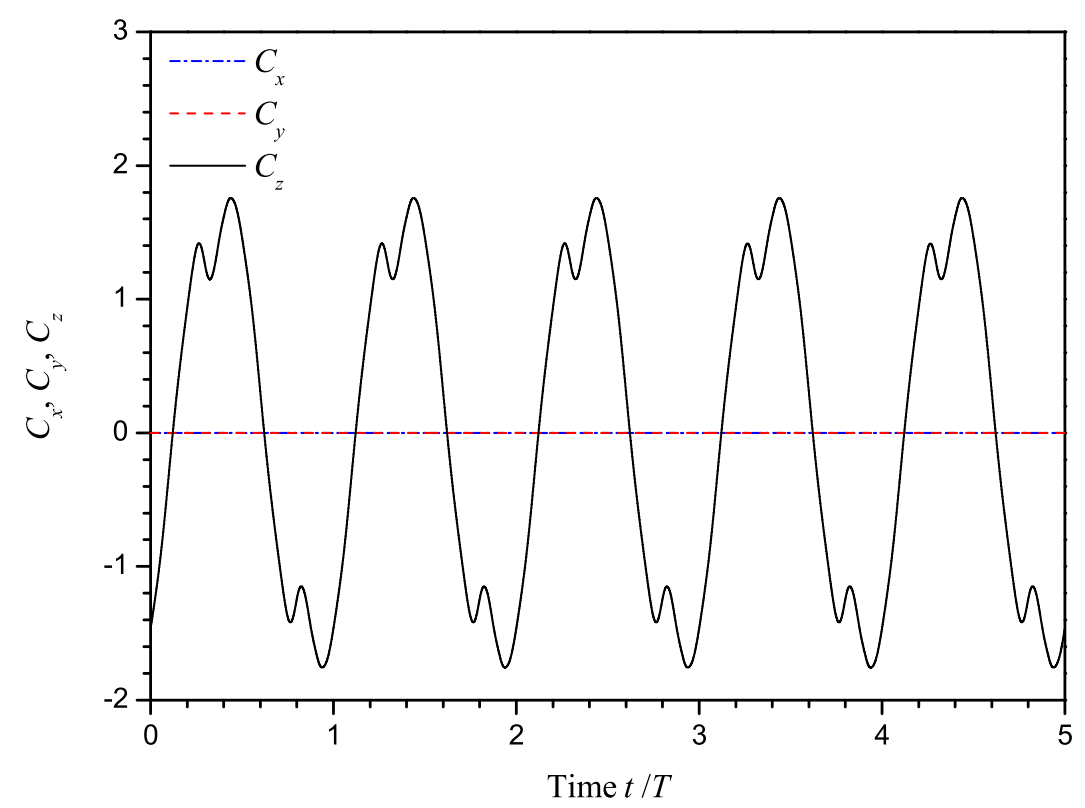

Figure 6 . Time traces of the force coefficients $C_{x}, C_{y}$ and $C_{z}$ of the disk over 5 oscillation cycles in the AS regime, where $R e=600$ and $K C=15$.

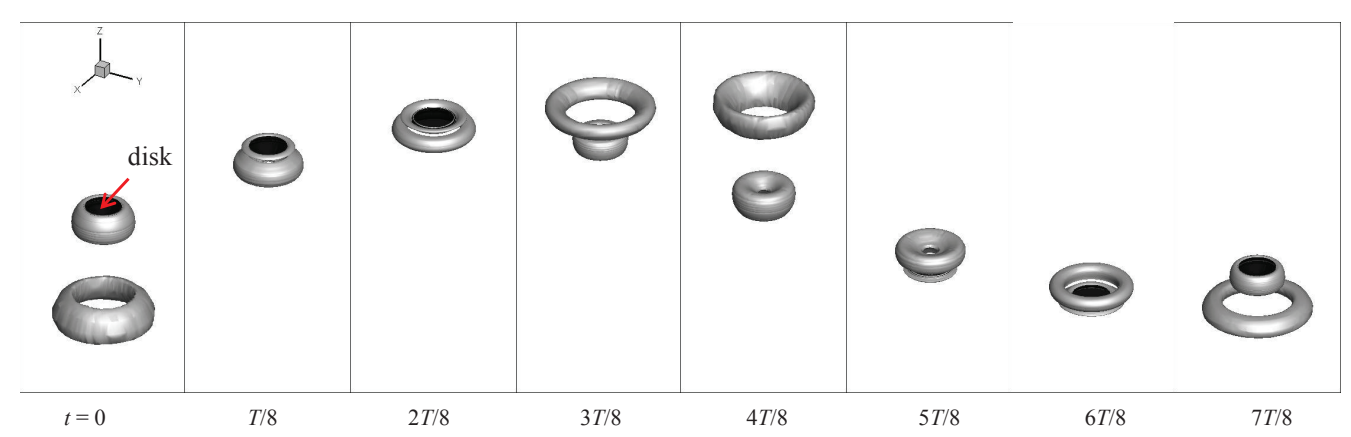

Figure 7. Vortical structures identified based on the $Q$-criterion during one oscillation cycle in the AS regime, where $R e=600$ and $K C=15$.

criterion for $R e=600$ and $K C=15$ in the AS regime. As shown in figure 7 , the flow is symmetric about the axis of the disk throughout the oscillation cycle. In the presented case, circular vortex rings are repeatedly generated with the periodic oscillations of the disk (see online supplementary material Movie 1 ). This vortex shedding results in oscillations in the time traces of $C_{z}$ at higher frequencies. As mentioned previously, the critical Reynolds number for the non-axisymmetric bifurcation of the uniform flow normal to a circular disk with $\chi=10$ has been reported to be $R e_{c 1} \approx 135$ (Shenoy \& Kleinstreuer 2008 ) and $R e_{c 1} \approx 129.6$ (Chrust et al. 2010). Although the Reynolds number in the case depicted in figure 7 is 600 , which is significantly higher than the reported $R e_{c 1}$ values, the flow in this oscillating case is still axisymmetric. One may argue that the Reynolds number defined for the oscillating case considered in this study is based on the maximum velocity of the disk $\left[U_{\max }\right.$, see equation (1.1)], thereby resulting in a nominally higher Reynolds number. However, if we calculate an average Reynolds number based on the average magnitude of the velocity of the oscillating disk, then this average Reynolds num- 

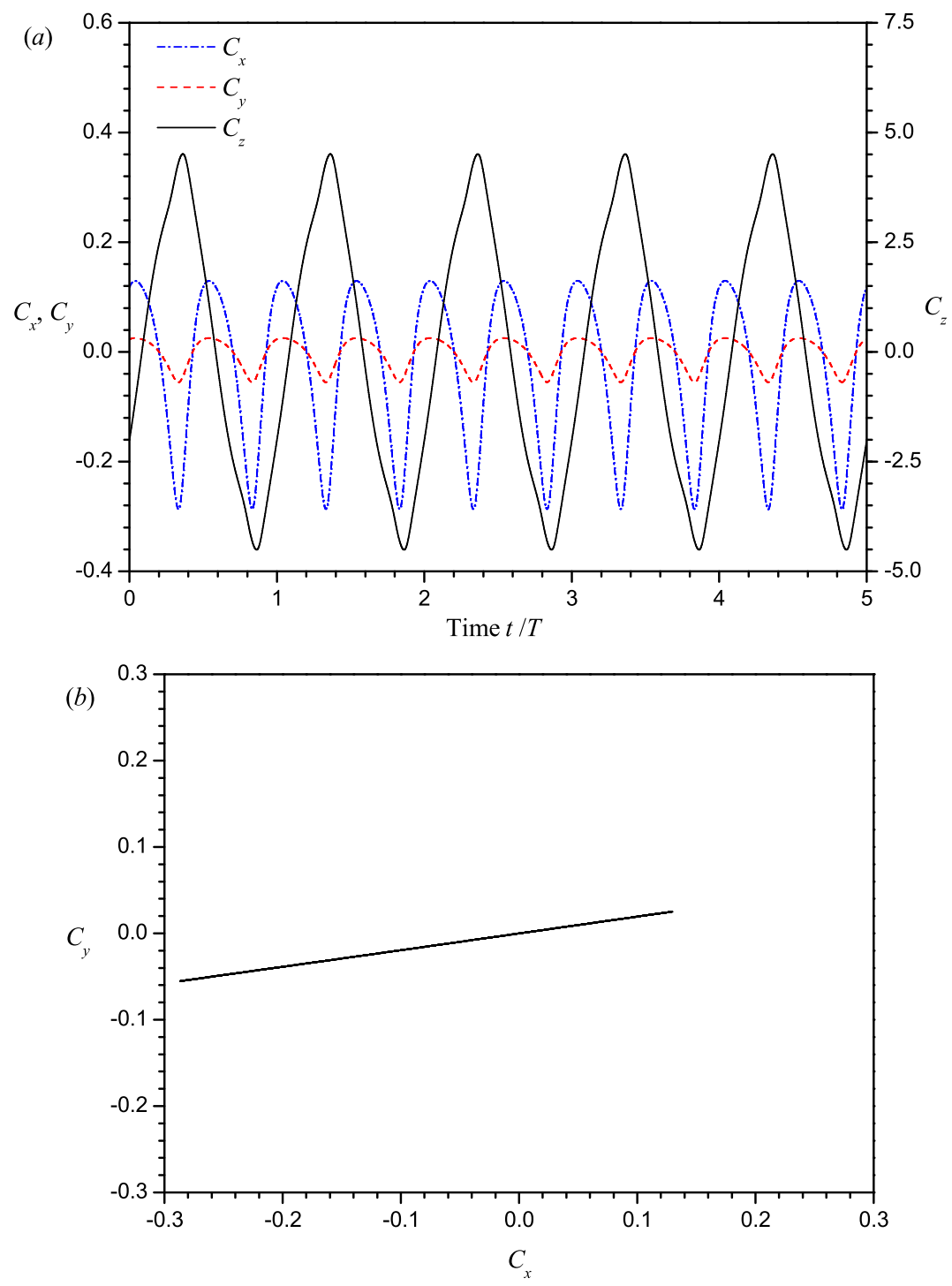

FIGURE 8. Force coefficient results for the disk over 5 oscillation cycles in the PSL regime, where $R e=411$ and $K C=2.57:(a)$ time traces of $C_{x}, C_{y}$ and $C_{z}$ and $(b)$ a phase-space plot of $C_{x}$ and $C_{y}$.

ber is $R e_{a v e}=382$, which is still significantly higher than the reported values of $R e_{c 1}$. This finding implies the existence of fundamental differences in fluid dynamics between a unidirectional flow and an oscillatory flow. Notably, the exactly circular vortical rings shown in figure 7 have not been observed in the wake of the uniform flow normal to a circular disk. This is because, for the uniform flow normal to a disk, the critical Reynolds number for the onset of vortex shedding is higher than the critical Reynolds number for non-axisymmetric bifurcation.

\subsection{Regime II: Planar symmetric flow in the low-KC region (PSL)}

Figure 8 shows the force coefficient results for the PSL regime, where $R e=411$ and $K C=$ 2.57. As shown in figure 8(a), the values of $C_{x}$ and $C_{y}$ are comparable to the value of $C_{z}$. 


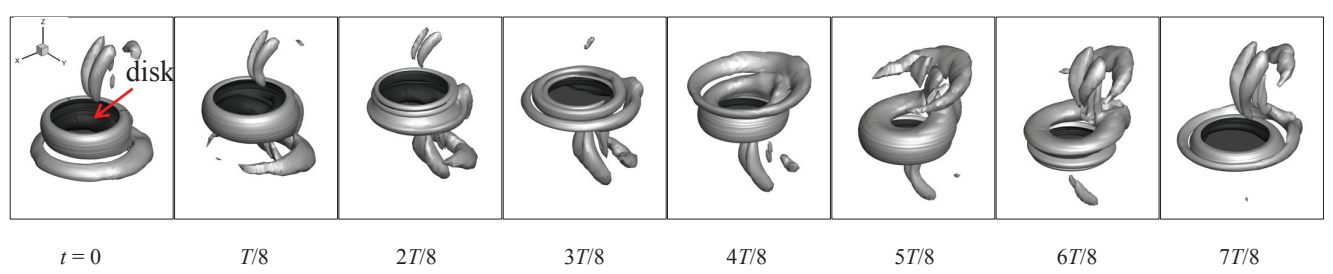

FiguRE 9. Vortical structures identified based on the $Q$-criterion during one oscillation cycle for the PSL regime, where $R e=411$ and $K C=2.57$.

The time traces of the force coefficients are periodic; however, the periods of $C_{x}$ and $C_{y}$ are one half of the period of $C_{z}$. This finding indicates that the transverse force oscillates twice during one oscillation cycle. It is evident that the noticeable transverse force acting on the disk arises because the flow surrounding the disk is no longer axisymmetric.

The vortical structures identified based on the $Q$-criterion for $R e=411$ and $K C=2.57$ in the PSL regime are shown in figure 9. As shown in figure 9, the flow around the disk in the PSL regime appears with a planar symmetric pattern rather than with an axisymmetric pattern. As the disk oscillates, vortex shedding initially begins along one side of the disk edge, and then, a crescent-moon-shaped vortex is generated, eventually resulting in a non-axisymmetric flow. Figure 9 shows that non-axisymmetric vortex shedding occurs twice during one oscillation cycle; accordingly, the transverse force coefficients $C_{x}$ and $C_{y}$ also oscillate twice in one cycle. A more detailed animation of the vortex shedding process has been included in the on-line supplementary material (Movie 2).

Figure $8(b)$ shows the phase-space plot of the $C_{x}$ and $C_{y}$ time traces shown in figure $8(a)$. The transverse force acting on the disk appears to oscillate back and forth along a fixed orientation, indicating that the flow is indeed planar symmetric and that the direction of the transverse force lies within the symmetry plane. The orientation of the symmetry plane is selected randomly. As shown in figure 10, the axial vorticity $\left(\omega_{z}\right)$ contour plots on several $z$ planes again demonstrate that the flow is planar symmetric. The present planar symmetric flow recalls the planar symmetric (or reflectionally symmetric) modes of the uniform flow normal to a circular disk at low Reynolds numbers, such as the "steady state" (SS) (Fabre et al. 2008), "standing wave" (SW) (Meliga et al. 2009) and "Zig-zig" (Zz) (Auguste et al. 2010) modes. Notably, the planar symmetric flow is one-sided with respect to the disk axis; see the flow observations in figures 9 and 10. This is because the vortex shedding locations on the two sides of the disk are always with the same orientation; see figure 9 . The resulting mean value of the transverse force acting on the disk is nonzero; see figure $8(b)$. For the uniform flow normal to a circular disk, a nonzero mean transverse force has also been observed in the SS and Zz modes.

Because a similar planar symmetric flow regime is observed in the high- $K C$ region (see $\S 3.4$ ), the present flow regime is referred to as the regime of planar symmetric flow in the low- $K C$ region (PSL).

\subsection{Regime III: Azimuthally rotating flow in the low-KC region ( $A R L)$}

Figure 11 shows the force coefficient results for the ARL regime, where $R e=800$ and $K C=2.5$. Compared with the time traces of the force coefficients in the PSL regime [see figure $8(a)$ ], the time traces of the force coefficients in the ARL regime [see figure 11 $(a)$ ] are similar overall except for the slight variation in the envelopes of $C_{x}$ and $C_{y}$. Figure $11(b)$ shows the phase-space plot of $C_{x}$ and $C_{y}$ over 64 oscillation cycles. A very slow rotation is observed in the transverse force. The arrow in figure $11(b)$ indicates the direction of rotation of the transverse force. It appears that the flow rotates slowly as 


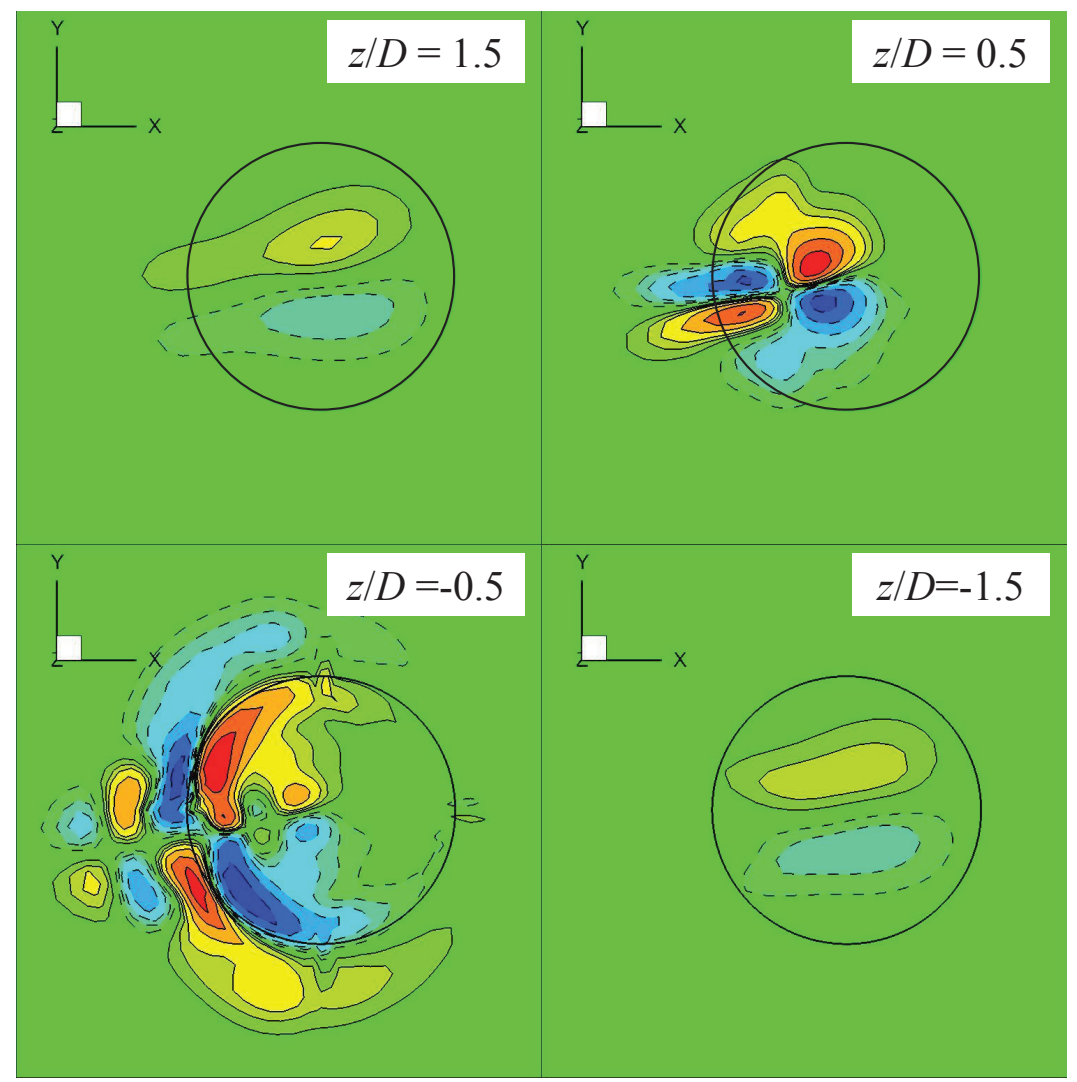

FIGURE 10. Contours of the axial vorticity $\left(\omega_{z}\right)$ on various $z$ planes for the PSL regime, where $R e=411, K C=2.57$ and $z_{d i s k}=0$. The circle is the projection of the disk. Positive and negative values of $\omega_{z}$ are represented by solid and dashed lines, respectively.

the disk passes through successive oscillations. Figure 12 shows the evolution of the axial vorticity $\left(\omega_{z}\right)$ contour on the plane $z / D=1$ when $z_{\text {disk }}=0$ with a time interval of $13 T$. It is observed that the flow is continuously rotating about the axis of the disk.

The vortical structures identified based on the $Q$-criterion for $R e=800$ and $K C=2.5$ in the ARL regime are shown in figure 13 and the on-line supplementary material (Movie 3 ). Although the rotational motion of the flow is very slow, the three-dimensional vortical structures in the ARL regime are more complicated than those in the PSL regime; see figure 9 . For the case of the flow normal to a circular disk, the flow begins to rotate about the axis of the disk after the secondary Hopf bifurcation. The planar symmetry observed in the previous "steady state" (SS) mode no longer holds. The flow exists in a "YinYang" (YY) state instead; see figure 5 in Shenoy \& Kleinstreuer (2008). However, the "Yin-Yang" mode observed in the uniform flow normal to a circular disk is not observed in the rotating flow considered here. As shown in figure 12, the instantaneous rotating flow is approximately planar symmetric, as observed in the PSL regime; see figure 10. This implies that the planar symmetric nature of the flow in the PSL regime is inherited in the ARL regime. Therefore, similar to the flow in the PSL regime, the flow in the ARL regime is also one-sided with respect to the disk axis, and this feature is again associated with a nonzero mean transverse force [see figure 11(b)].

Because a similar azimuthal rotating flow regime is observed in the high- $K C$ region 

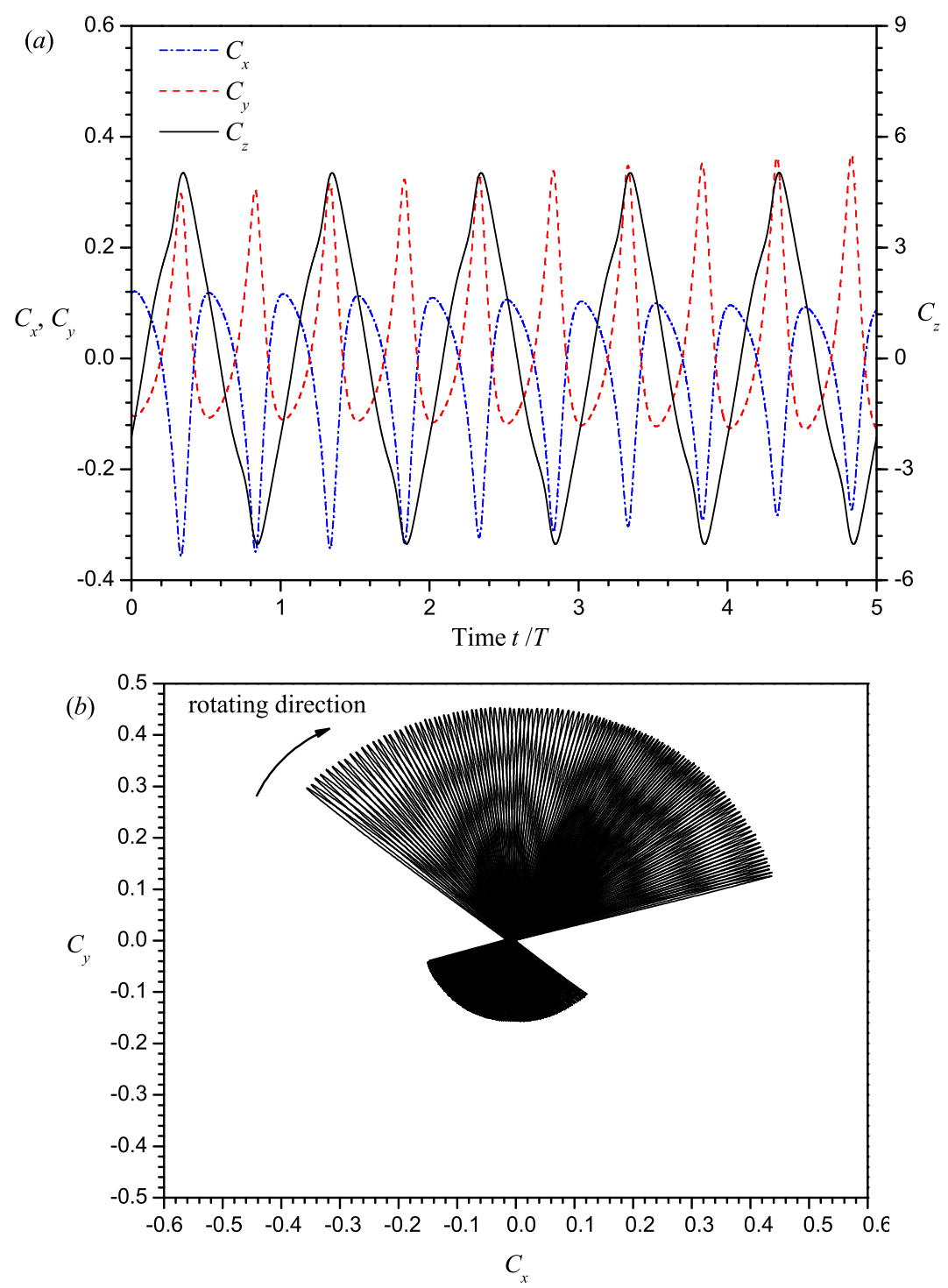

FiguRE 11. Force coefficient results for the disk in the ARL regime, where $R e=800$ and $K C=2.5:(a)$ time traces of $C_{x}, C_{y}$ and $C_{z}$ over 5 oscillation cycles and $(b)$ a phase-space plot of $C_{x}$ and $C_{y}$ over 64 oscillation cycles.

(see $\S 3.5$ ), the present flow regime is referred to as the regime of azimuthal rotating flow in the low- $K C$ region (ARL).

\subsection{Regime IV: Planar symmetric flow in the high-KC region (PSH)}

Figure 14 shows the force coefficient results for the PSH regime, where $R e=500$ and $K C=20$. As shown in figure $14(a)$, the values of $C_{x}$ and $C_{y}$ are comparable to the value of $C_{z}$, indicating a non-axisymmetric flow. Similar to the PSL regime (see figure $8 a$ ), the time traces of the force coefficients are periodic and the periods of $C_{x}$ and $C_{y}$ are one half of the period of $C_{z}$. A high-frequency fluctuation is evident in $C_{z}$. This is because 


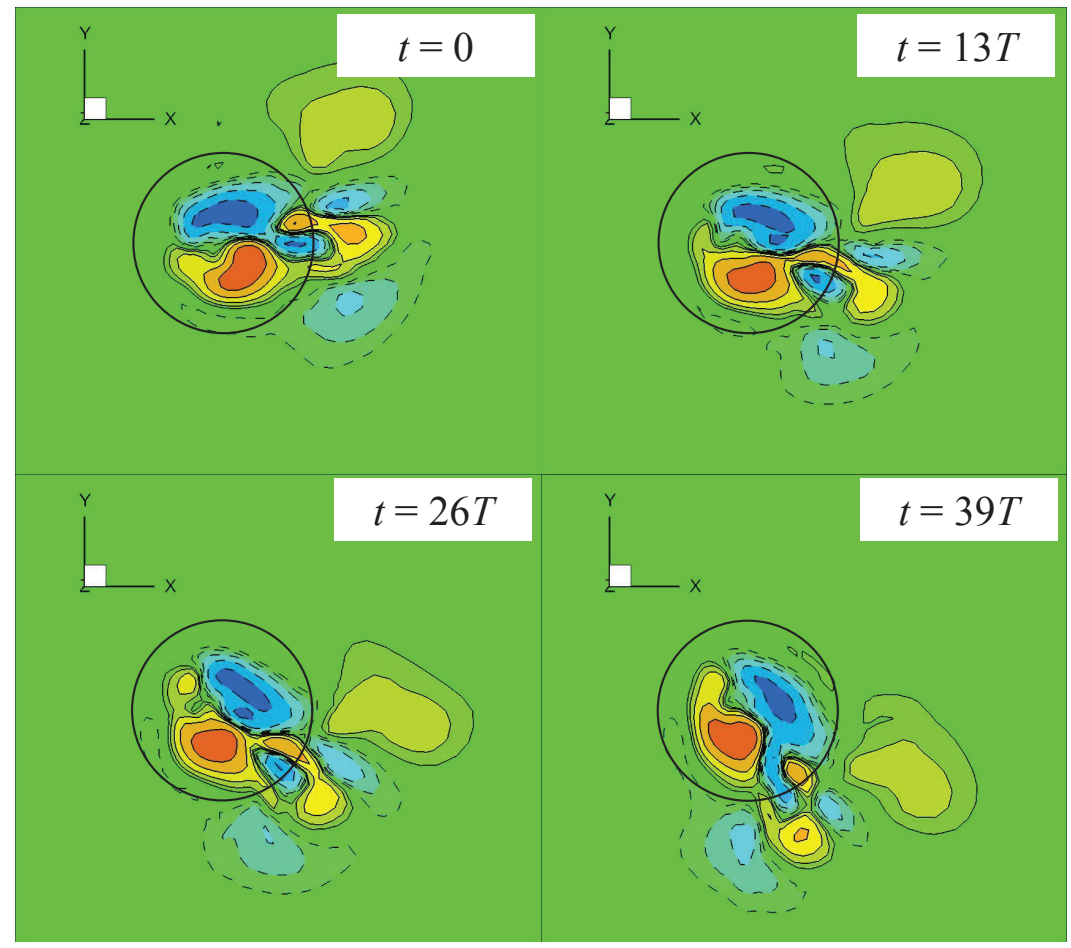

FIGURE 12. Evolution of the axial vorticity $\omega_{z}$ on the $z / D=1$ plane for the ARL regime, where $R e=800, K C=2.5$ and $z_{d i s k}=0$. The circle is the projection of the disk. Positive and negative values of $\omega_{z}$ are represented by solid and dashed lines, respectively.

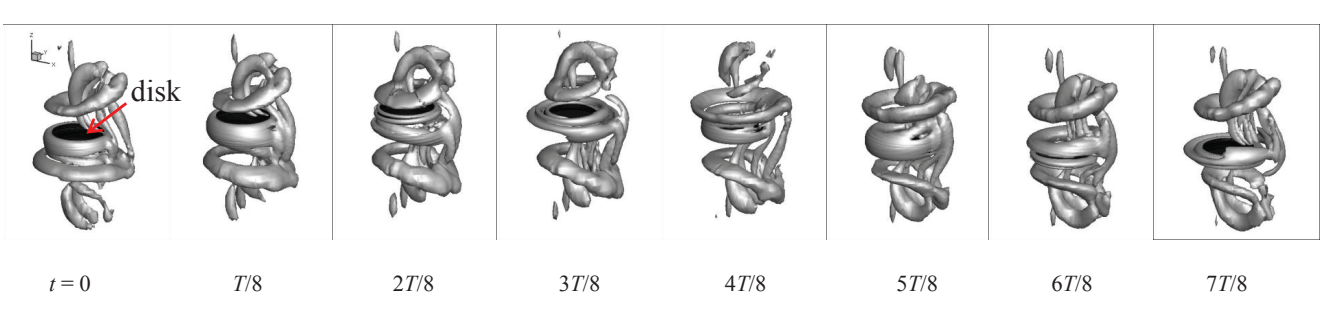

FIGURE 13. Vortical structures identified based on the $Q$-criterion during one oscillation cycle for the ARL regime, where $R e=800$ and $K C=2.5$.

the vortex shedding along the disk occurs more frequently in this high- $K C$ case than that in low- $K C$ cases.

Figure 14(b) shows the phase-space plot of $C_{x}$ and $C_{y}$ for $R e=500$ and $K C=20$ in the PSH regime. As observed in the PSL regime (see figure $8 b$ ), the transverse force acting on the disk in the PSH regime has a fixed orientation and a nonzero mean value, indicating a one-sided planar symmetric flow. The transverse force acting on the disk oscillates within the plane of symmetry.

The vortical structures identified based on the $Q$-criterion for the case with $R e=500$ and $K C=20$ in the PSH regime are shown in figure 15. For the cases at high $K C$, the disk travels a farther distance and the vortical structures behind the disk are more adequately developed. Therefore, apparent hairpin vortices are observed in figure 15 . However, the vortices are shed in a fixed azimuthal direction with respect to the disk, unlike the double-sided vortex shedding mode observed in the "standing wave" (SW) 

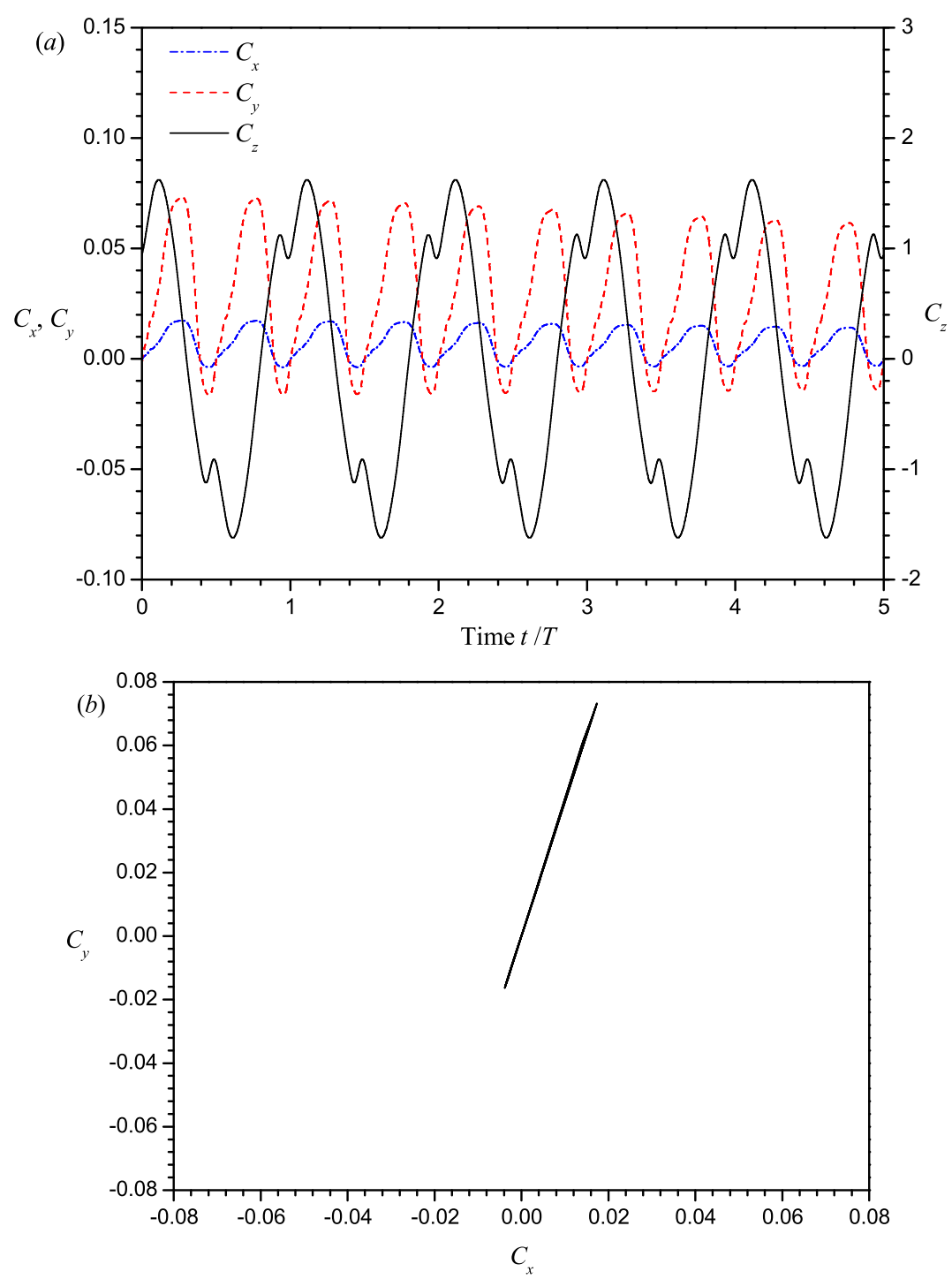

FiguRE 14. Force coefficient results for the disk over 5 oscillation cycles in the PSH regime, where $R e=500$ and $K C=20:(a)$ time traces of $C_{x}, C_{y}$ and $C_{z}$ and $(b)$ a phase-space plot of $C_{x}$ and $C_{y}$.

mode for the flow normal to a circular disk; see figure 8 in Shenoy \& Kleinstreuer (2008). Figure 16 shows the contours of the axial vorticity $\left(\omega_{z}\right)$ on various $z$ planes for the case of $R e=500, K C=20$ and $z_{\text {disk }}=0$. The findings again indicate that the flow is planar symmetric. A more detailed animation of the vortex shedding process has been included in the on-line supplementary material (Movie 4). In consideration of the planar symmetric flow regime observed in the low- $K C$ region (the PSL regime), as presented in $\S 3.2$, the present regime is referred to as the regime of planar symmetric flow in the high- $K C$ region $(\mathrm{PSH})$. 


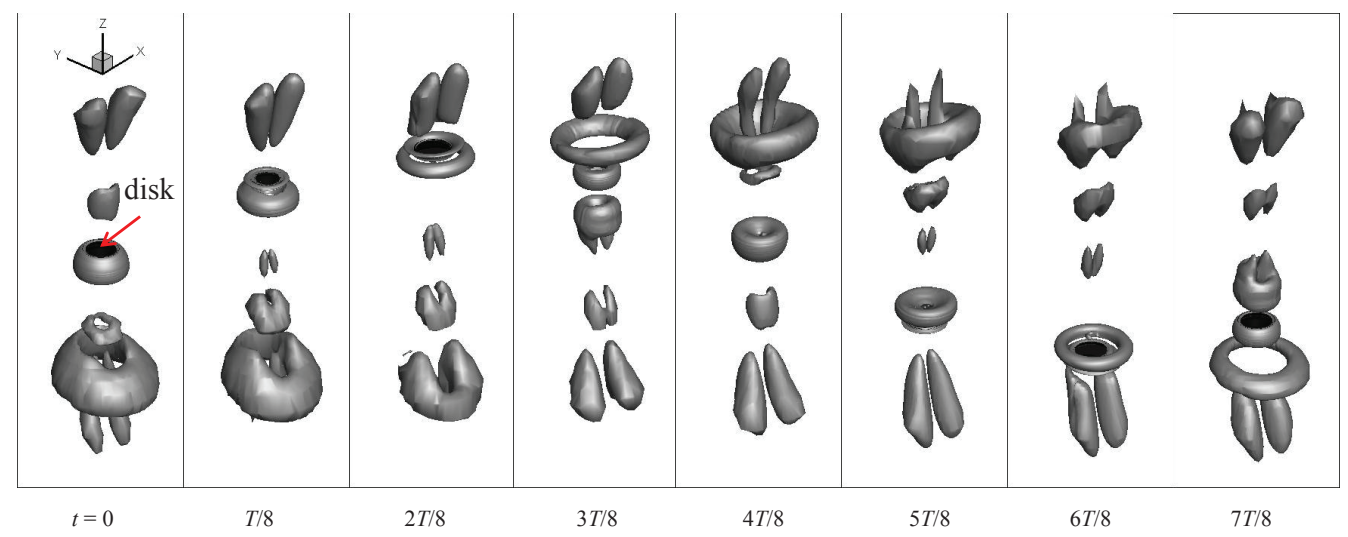

FigURE 15. Vortical structures identified based on the $Q$-criterion during one oscillation cycle for the PSH regime, where $R e=500$ and $K C=20$.

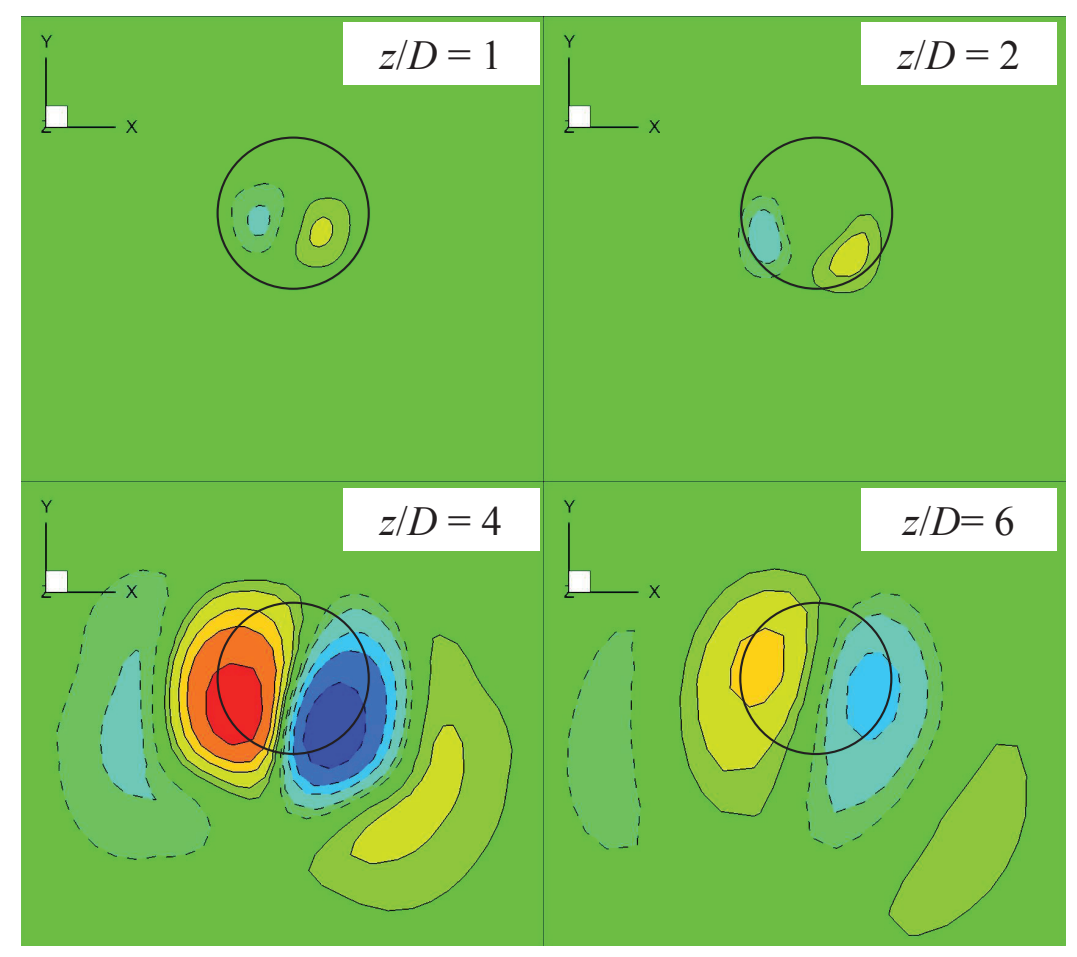

Figure 16. Contours of the axial vorticity $\left(\omega_{z}\right)$ on various $z$ planes for the PSH regime, where $R e=500, K C=20$ and $z_{d i s k}=0$. The circle is the projection of the disk. Positive and negative values of $\omega_{z}$ are represented by solid and dashed lines, respectively.

\subsection{Regime $V$ : Azimuthally rotating flow in the high- $K C$ region ( $A R H$ )}

Figure 17 shows the force coefficient results for the ARH regime, where $R e=600$ and $K C=24$. As shown in figure $17(a), C_{z}$ varies periodically, whereas $C_{x}$ and $C_{y}$ exhibit a slow variation in magnitude. The phase-space plot of $C_{x}$ and $C_{y}$ shown in figure $17(b)$ indicates a rotational motion of the flow. Compared with the rotational motion observed in the ARL regime (see figure 11b), the rotational motion seen in this case is much faster. Figure 18 shows the evolution of the axial vorticity $\left(\omega_{z}\right)$ contours on the $z / D=4$ plane 

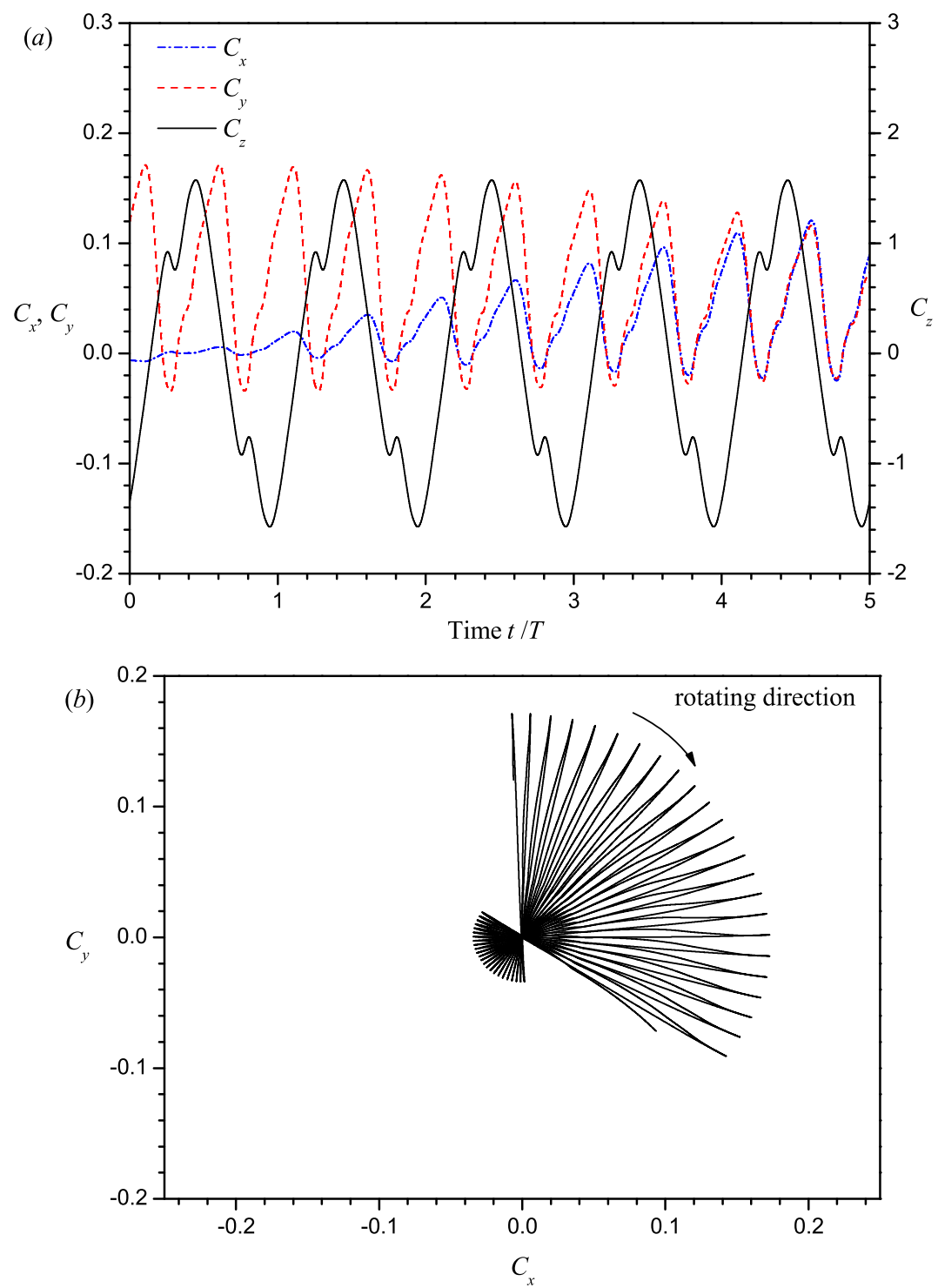

FiguRE 17. Force coefficient results for the disk in the $\mathrm{ARH}$ regime, where $R e=600$ and $K C=24:(a)$ time traces of $C_{x}, C_{y}$ and $C_{z}$ over 5 oscillation cycles and $(b)$ a phase-space plot of $C_{x}$ and $C_{y}$ over 12 oscillation cycles.

when $z_{\text {disk }}=0$ with a time interval of $2 T$. It is observed that the flow is continuously rotating about the axis of the disk.

Figure 19 shows the variations in the vortical structures identified based on the $Q$ criterion during one oscillation cycle for $R e=600$ and $K C=24$ in the ARH regime. The observed flow structures are more disordered than those in the PSH regime; see figure 15 and the on-line supplementary material (Movie 5). This is because the Reynolds number in the ARH regime is higher than that in the PSH regime. Hairpin vortical structures are clearly observed in figure 19, as in the wake of the uniform flow normal to a disk. It is again observed that the vortical structures are one-sided with respect to the axis of the disk and that the mean transverse force acting on the disk is nonzero; see figure $17(b)$. 


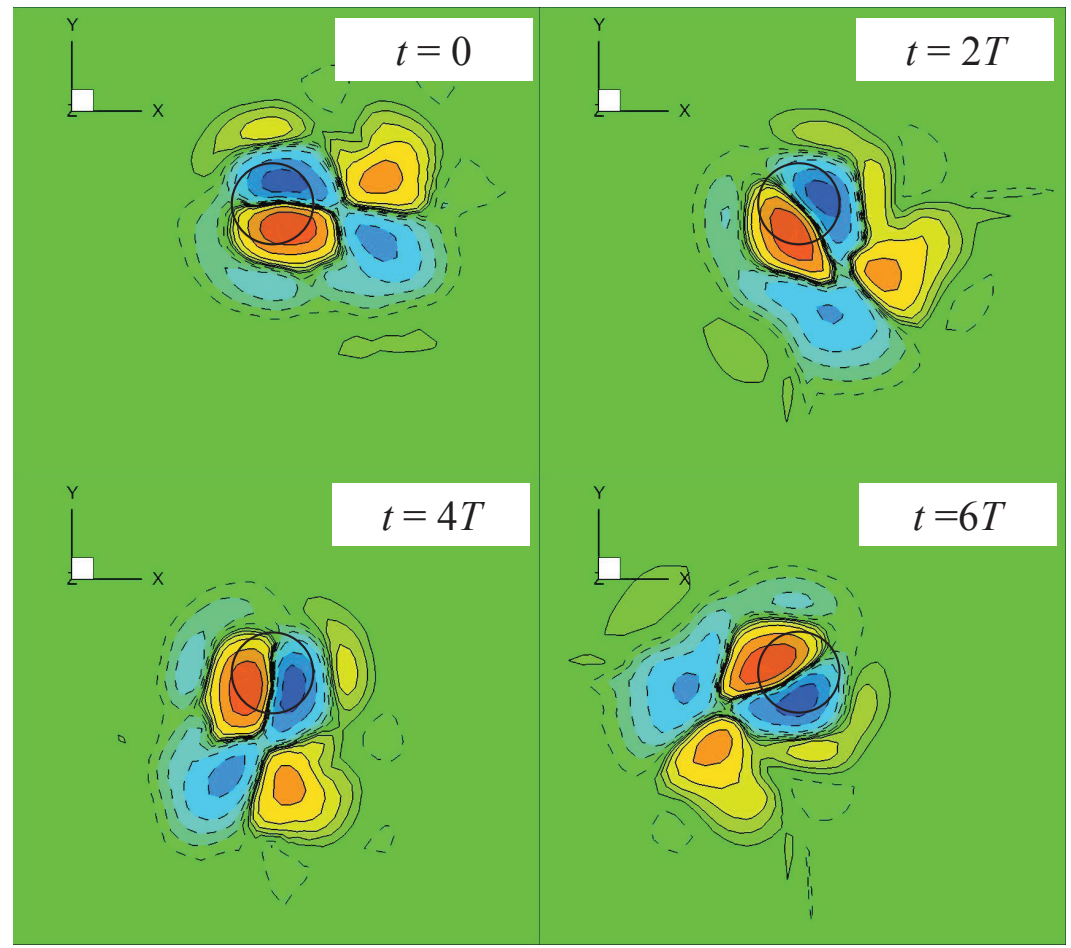

FiguRE 18. Evolution of the axial vorticity $\omega_{z}$ on the plane $z / D=4$ for the ARH regime, where $R e=600, K C=24$ and $z_{d i s k}=0$. The circle is the projection of the disk. Positive and negative values of $\omega_{z}$ are represented by solid and dashed lines, respectively.

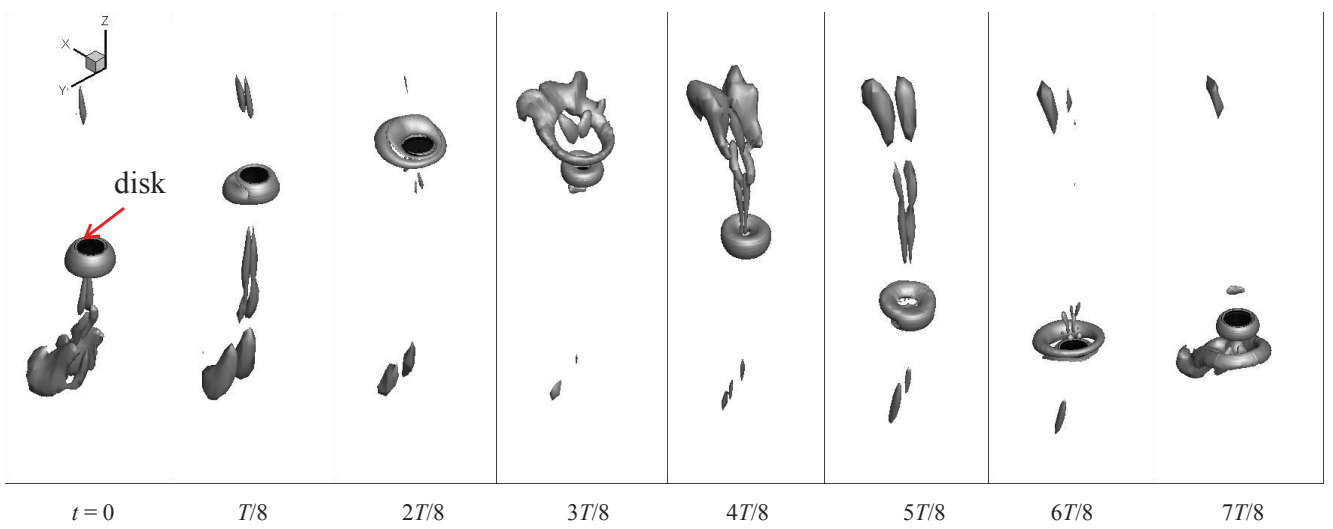

FiguRE 19. Vortical structures identified based on the $Q$-criterion during one oscillation cycle for the ARH regime, where $R e=600$ and $K C=24$.

In consideration of the azimuthal rotating flow regime observed in the low- $K C$ region (the ARL regime), as discussed in $\S 3.3$, the present regime is referred to as the regime of azimuthally rotating flow in the high- $K C$ region $(\mathrm{ARH})$.

\subsection{Discussions on the steady and oscillatory flow regimes}

Recalling the bifurcation scenarios in the wake of the uniform flow normal to a thin disk (see $\S 1$ ), several flow regimes are revealed successively as Re increases: "steady axisym- 
metric state" (Auguste et al. 2010), "steady asymmetric state" Shenoy \& Kleinstreuer (2008), "reflectional symmetry breaking" (Fabre et al. 2008), and later some more complicated states depending on the aspect ratio of the disk. It is noted that all these states are used for describing the "steady-flow" situation with a constant Re. For the oscillating disk case, the "steady-flow" situation is never held, instead, the actual Reynolds number increases and decreases as the disk oscillates. The saturated "steady-flow" state at a particular $R e$ is not able to be achieved in the oscillating case. Moreover, compared with the "steady-flow" case, the oscillating disk moves back and forth periodically, the disk may go into its own wake. However, it does not mean that the oscillating disk case is not comparable to the "steady-flow" case. A sufficiently large $K C$ of an oscillating disk provides a large distance for the evolution of wake flow, therefore giving us an opportunity to catch some scenario fragments which are similar to those "steady-flow" states.

In this study, the range of $K C$ is up to 24 . At the lower part of figure 5 , the regimes PSL and ARL are in the range of $2.5<K C<7$, approximately, corresponding to an oscillatory amplitude of $0.4 D<a<1.4 D$. This amplitude is too small to have a developed wake flow, as confirmed in figures 9 and 13 and Movies 2 and 3 in the on-line supplementary material. At the upper part of figure 5, the value of $K C$ in the regimes PSH and ARH is up to 24, corresponding to an oscillatory amplitude of $a \approx 3.82 D$. This distance allows a wake evolution as that in the "steady-flow" case. As shown in the case of $R e=500$ and $K C=20$ (see figure 15), the flow is planar symmetric as described in $\S 3.4$. At the topmost and lowest locations (see the pictures at $t=2 T / 8$ and $6 T / 8$ respectively in figure 15$)$, the actual instantaneous Reynolds number is very small. Therefore, the toroidal vortex is generated approximately. This could be interpreted as a co-existence of different "steady-flow" states in the oscillating disk case, see also Movie 4 in the on-line supplementary material. For the case of $R e=600$ and $K C=24$ (see figure 19), the increase in Reynolds number results in a new regime corresponding to the "reflectional symmetry breaking state" in the "steady-flow" case (Fabre et al. 2008).

It appears that there are some connections between the oscillating disk at a large $K C$ and the "steady-flow" case. However, it must be kept in mind that the oscillating disk case presented here are intrinsically different with the "steady-flow" situation. In other words, the $K C=24$ is still too small to be considered as a "sufficiently large" $K C$ at which those bifurcations in the "steady-flow" case could be observed in one oscillating cycle.

\section{Concluding remarks}

The flow around an oscillating circular disk was investigated using the DNS approach. The disk was forced to oscillate sinusoidally along its axis. The aspect ratio (diameter/thickness) of the disk was 10. The ranges considered for the Reynolds number $(R e)$ and the Keulegan-Carpenter number $(K C)$ were $50 \leqslant R e \leqslant 800$ and $1 \leqslant K C \leqslant 24$, respectively. In the considered $R e-K C$ space, one axisymmetric flow regime and two non-axisymmetric flow regimes were identified. In each non-axisymmetric flow regime, the flow was planar symmetric at lower Reynolds numbers and began to rotate about the axis of the disk as the Reynolds number increased. Therefore, five flow regimes were identified: (I) axisymmetric flow (AS), (II) planar symmetric flow in the low- $K C$ region (PSL), (III) azimuthally rotating flow in the low- $K C$ region (ARL), (IV) planar symmetric flow in the high- $K C$ region $(\mathrm{PSH})$ and $(\mathrm{V})$ azimuthally rotating flow in the high- $K C$ region $(\mathrm{ARH})$. In the non-axisymmetric flow regimes, the flow around the disk was found to be one-sided with respect to the axis of the disk and associated with a nonzero mean value of the transverse force acting on the disk. 

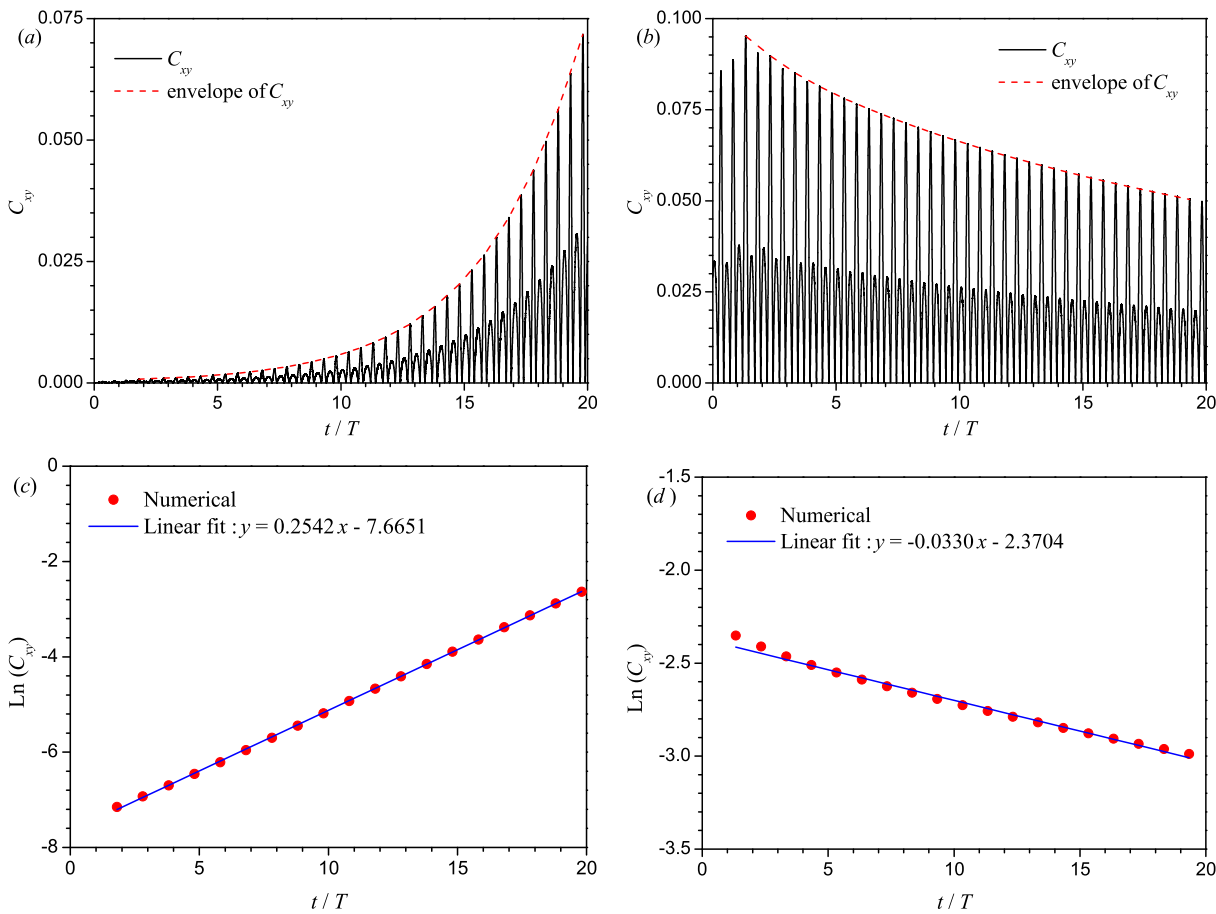

FiguRE 20. Determination of the growth/decay rates of the amplitude of $C_{x y}$ for the cases based on a perturbed initial condition. The time traces of $C_{x y}$ for the cases of $(a)(R e, K C)=(300,3)$ and $(b)(R e, K C)=(300,2.5),(c)$ and $(d)$ the corresponding exponential fit of the envelop of $C_{x y}$.

It should be noted that the critical boundaries between the different flow regimes in the $R e-K C$ space were determined based on a limited number of numerical simulations. More accurate boundaries could be obtained through more extensive numerical simulations. Because only one disk aspect ratio, namely, $\chi=10$, was considered in this study, further research is planned to study the effects of the aspect ratio on the flow regimes and the critical boundaries between different flow regimes.

The authors would like to thank the support from Shanghai Yang Fan Program (Grant no. 15YF1406100), the National Natural Science Foundation of China (Grant nos. 51509152, 11632011 and 51239007) and the Newton Research Collaboration Programme (Grant no. NRCP/1415/211). The numerical simulations were supported by Center for HPC, Shanghai Jiao Tong University. The authors acknowledge the constructive comments of the reviewers which indeed improved the quality of this paper.

\section{Appendix A}

This appendix provides additional information of the procedures used to determine the accurate critical $R e$ and $K C$ numbers for the axisymmetric and non-axisymmetric bifurcations. The threshold is determined roughly based on the extensive simulations at various $R e$ and $K C$ as indicated by the solid dots in figure 5 . It is observed that the threshold lays between the axisymmetric case $(R e, K C)=(300,2.5)$ and the nonaxisymmetric case $(R e, K C)=(300,3)$. Now we use a slightly perturbed flow as the initial condition for the case $(R e, K C)=(300,3)$. As shown in figure $20(a)$, a growth of 


$\begin{array}{cccccc}(R e, K C) & (R e, K C) & (R e, K C) & (R e, K C) & (R e, K C) & (R e, K C) \\ & & & & & \\ (178.61,5) & (200,8.40) & (300,5.63) & (400,4.70) & (500,6.01) & (600,5.58) \\ (700,4.96) & (800,4.49) & (200,3.91) & (300,2.56) & (400,2.07) & (500,2.01) \\ (600,1.99) & (700,1.41) & (800,1.00) & (300,22.65) & (400,13.3) & (500,15.79) \\ (600,19.28) & (700,19.29) & (800,19.37) & (284.12,24) & & \end{array}$

TABLE 4. Critical Re and $K C$ numbers for the axisymmetric and non-axisymmetric bifurcations.

the amplitude of the transverse force coefficient $C_{x y}$ is observed. By fitting the envelope of the time traces of $C_{x y}$ to an exponential function of time, a growth rate of 2.2542 (see figure 20c) is determined (Ghidersa \& Dušek 2000; Bohorquez et al. 2011). Similarly, the case of $(R e, K C)=(300,2.5)$ is conducted with the solution for the case of $(R e, K C)=$ $(300,3)$ as an initial condition. Then, a decay of the amplitude of $C_{x y}$ is observed and the decay rate is determined to be -0.0330 (see figures $20 b$ and $20 d$ ). By linear interpolation of the amplification rates, the threshold is obtained at $(R e, K C)=(300,2.56)$. Similar procedures are used to determine to the accurate threshold at other locations. In this study, 22 sets of simulations are performed and the determined critical $R e$ and $K C$ numbers are shown in table 4 .

\section{REFERENCES}

An, H., Cheng, L. \& Zhao, M. 2011 Direct numerical simulation of oscillatory flow around a circular cylinder at low Keulegan-Carpenter number. J. Fluid Mech. 666, 77-103.

Auguste, F., Fabre, D. \& Magnaudet, J. 2010 Bifurcations in the wake of a thick circular disk. Theor. Compu. Fluid Dyn. 24 (1), 305-313.

Auguste, F., Magnaudet, J. \& Fabre, D. 2013 Falling styles of disks. J. Fluid Mech. 719, 388-405.

Bearman, P. W. 1984 Vortex shedding from oscillating bluff bodies. Ann. Rev. Fluid Mech. 16 (1), 195-222.

Berger, E., Scholz, D. \& Schumm, M. 1990 Coherent vortex structures in the wake of a sphere and a circular disk at rest and under forced vibrations. J. Fluids Struct. 4 (3), 231-257.

Bohorquez, P., Sanmiguel-rojas, E., Sevilia, A., Jiménez-gonzález, J. I. \& MartínezBAZÁN, C. 2011 Stability and dynamics of the laminar wake past a slender blunt-based axisymmetric body. J. Fluid Mech. 676, 110-144.

Chrust, M., Bouchet, G. \& DušEk, J. 2010 Parametric study of the transition in the wake of oblate spheroids and flat cylinders. J. Fluid Mech. 665, 199-208.

Chrust, M., Bouchet, G. \& DušEK, J. 2014 Effect of solid body degrees of freedom on the path instabilities of freely falling or rising flat cylinders. J. Fluids Struct. 47, 55-70.

Chrust, M, Dauteuille, C, Bobinski, T, Rokicki, J, Goujon-Durand, S, Wesfreid, Je, Bouchet, G \& Dušek, J 2015 Effect of inclination on the transition scenario in the wake of fixed disks and flat cylinders. J. Fluid Mech. 770, 189-209.

Ern, P., Risso, F., Fabre, D. \& Magnaudet, J. 2012 Wake-induced oscillatory paths of bodies freely rising or falling in fluids. Ann. Rev. Fluid Mech. 44, 97-121.

Fabre, D., Auguste, F. \& Magnaudet, J. 2008 Bifurcations and symmetry breaking in the wake of axisymmetric bodies. Phys. Fluids 20, 051702.

Fernandes, P. C., Risso, F., Ern, P. \& Magnaudet, J. 2007 Oscillatory motion and wake instability of freely rising axisymmetric bodies. J. Fluid Mech. 573, 479-502.

GhidersA, B. \& DušEK, J. 2000 Breaking of axisymmetry and onset of unsteadiness in the wake of a sphere. J. Fluid Mech. 423, 33-69.

Hunt, J. C. R., Wray, A. A. \& Moin, P. 1988 Eddies, streams, and convergence zones in 
turbulent flows. In Center for Turbulence Research Report CTR-S88, pp. 193-208. USA: NASA.

Kuo, Y. H. \& Baldwin, L. V. 1967 The formation of elliptical wakes. J. Fluid Mech. 27 (02), $353-360$.

Marshall, D. \& Stanton, T. E. 1931 On the eddy system in the wake of flat circular plates in three dimensional flow. Proceedings of the Royal Society of London. Series A, Containing Papers of a Mathematical and Physical Character 130 (813), 295-301.

Meliga, P., Chomaz, J. M. \& Sipp, D. 2009 Global mode interaction and pattern selection in the wake of a disk: a weakly nonlinear expansion. J. Fluid Mech. 633, 159-189.

Michael, P. 1966 Steady motion of a disk in a viscous fluid. Phys. Fluids 9, 466.

Morison, J. R., O’Brien, M. P., Johnson, J. W. \& SchaAf, S. A. 1950 The force exerted by surface waves on piles. Petrol. Trans. 189, 149-157.

Natarajan, Ramesh \& Acrivos, Andreas 1993 The instability of the steady flow past spheres and disks. J. Fluid Mech. 254, 323-344.

OpenFOAM 2009 The Open Source CFD Toolbox, Programmer's Guide, Version 1.6. Boston, MA, USA: OpenCFD Limited.

Rimon, Y. 1969 Numerical solution of the incompressible time-dependent viscous flow past a thin oblate spheroid. Phys. Fluids 12, II-65-75.

Rivet, J. P., Henon, M., Frisch, U. \& D'Humieres, D. 1988 Simulating fully threedimensional external flow by lattice gas methods. Europhys. Letters 7, 231.

Roberts, J. B. 1973 Coherence measurements in an axisymmetric wake. AIAA J. 11, 15691571.

Roos, F. W. \& Willmarth, W. W. 1971 Some experimental results on sphere and disk drag. AIAA J. 9, 285-291.

Shenoy, A. R. \& Kleinstreuer, C. 2008 Flow over a thin circular disk at low to moderate Reynolds numbers. J. Fluid Mech. 605, 253-262.

Shenoy, A. R. \& Kleinstreuer, C. 2010 Influence of aspect ratio on the dynamics of a freely moving circular disk. J. Fluid Mech. 653, 463-487.

Tchoufag, J., Fabre, D. \& Magnaudet, J. 2014 Global linear stability analysis of the wake and path of buoyancy-driven disks and thin cylinders. J. Fluid Mech. 740, 278-311.

Weller, H. G., Tabor, G., Jasak, H. \& Fureby, C. 1998 A tensorial approach to computational continuum mechanics using object-oriented techniques. Comput. Phys. 12, 620-631.

Williamson, C. H. K. \& Govardhan, R. 2008 A brief review of recent results in vortexinduced vibrations. J. Wind Eng. Ind. Aerodyn. 96, 713-735.

Willmarth, W. W., Hawk, N. E. \& Harvey, R. L. 1964 Steady and unsteady motions and wakes of freely falling disks. Phys. Fluids .

YAng, J., Liu, M., Wu, G., Liu, Q. \& Zhang, X. 2015 Low-frequency characteristics in the wake of a circular disk. Phys. Fluids 27 (6), 064101.

Yang, J., Liu, M., Wu, G., Zhong, W. \& Zhang, X. 2014a Numerical study on coherent structure behind a circular disk. J. Fluids Struct. 51, 172-188.

YANG, J., TIAN, X. \& LI, X. $2014 b$ Hydrodynamic characteristics of an oscillating circular disk under steady in-plane current conditions. Ocean Eng. 75, 53-63.

YANG, J., Wu, G., Zhong, W. \& Liu, M. 2014c Numerical study on bifurcations in the wake of a circular disk. Int. J. Comput. Fluid Dyn. 28 (5), 187-203.

Zhong, H. J. \& Lee, C. B. 2012 The wake of falling disks at low Reynolds numbers. Acta Mech. Sin. 28, 1-5. 